\title{
A Line Search Exact Penalty Method Using Steering Rules
}

\author{
Richard H. Byrd ${ }^{\dagger} \quad$ Gabriel Lopez-Calva ${ }^{\ddagger} \quad$ Jorge Nocedal ${ }^{\S}$ \\ January 11, 2009
}

\begin{abstract}
Line search algorithms for nonlinear programming must include safeguards to enjoy global convergence properties. This paper describes an exact penalization approach that extends the class of problems that can be solved with line search SQP methods. In the new algorithm, the penalty parameter is adjusted at every iteration to ensure sufficient progress in linear feasibility and to promote acceptance of the step. A trust region is used to assist in the determination of the penalty parameter (but not in the step computation). It is shown that the algorithm enjoys favorable global convergence properties. Numerical experiments illustrate the behavior of the algorithm on various difficult situations.
\end{abstract}

\section{Introduction}

Exact penalty methods have proved to be effective techniques for solving difficult nonlinear programs. They overcome the difficulties posed by inconsistent constraint linearizations [13] and are successful in solving certain classes of problems in which standard constraint qualifications are not satisfied $[2,3,22,20,10,18]$. Despite their appeal, it has proved difficult to design penalty methods that perform well over a wide range of problems; the main difficulty lies in choosing appropriate values of the penalty parameter. Various approaches proposed in the literature update the penalty parameter only if convergence to an undesirable point appears to be taking place; see e.g. [28, 19] and the references therein. This can result in inefficient behavior and requires heuristics to determine when to change the penalty parameter.

Recently, a new strategy for updating the penalty parameter has been proposed in the context of trust region methods $[6,8]$. In that approach, the penalty parameter is selected

\footnotetext{
${ }^{\dagger}$ Department of Computer Science, University of Colorado, Boulder, CO, 80309. This author was supported by National Science Foundation grant CMMI 0728190.

${ }^{\ddagger}$ Department of Industrial Engineering and Management Sciences, Northwestern University, Evanston, IL, 60208. This author was supported by Department of Energy grant DE-FG02-87ER25047-A004.

${ }^{\S}$ Department of Electrical Engineering and Computer Science, Northwestern University, Evanston, IL, 60208. This author was supported by National National Science Foundation grants CCR-0219438 and CCF0514772 .
} 
at every iteration so that sufficient progress toward feasibility and optimality is guaranteed, to first order. This requires that an auxiliary subproblem (a linear program) be solved in certain cases. A particular implementation of this approach has been incorporated into the active-set method of the KNITRO [9, 30] package and has proved to be effective in practice. The technique just mentioned relies on the fact that the optimization algorithm is of the trust region kind.

In this paper we describe line search penalty methods for nonlinear programming. Unlike trust region methods, which control the quality and length of the steps, line search methods can produce very large and unproductive search directions in the neighborhood of points where standard regularity conditions are not satisfied, and this situation can lead to failures that would not occur with a trust region method. By relaxing the constraints and ensuring that steady progress toward the solution is made, the proposed method enjoys the same type of global convergence properties as trust region methods. The global analysis thus shows that use of exact penalty methods can have a regularizing effect without a trust region or an explicit regularization term.

To achieve these goals, the algorithm solves a linear program with an auxiliary trust region that helps determine the adequacy of the penalty parameter. The algorithm is nevertheless a pure line search method because the step computation does not depend on the auxiliary trust region - only the choice of the penalty parameter depends on it. In fact the the auxiliary trust region radius may be fixed at an arbitrary value without affecting convergence properties.

The new algorithm incurs an additional cost compared with classical line search methods. At those iterations in which the penalty parameter must be adjusted, an auxiliary linear program must be solved and the SQP step must be recomputed one or more times using larger values of the penalty parameter. This extra cost may, however, not be significant because warms starts can be employed in the solution of these additional quadratic programs. Furthermore, the hope is that the new strategy yields savings in iterations and improves the robustness of the method. An attractive feature of the new algorithm is that it treats all problems (regular or deficient) equally and does not need to resort to special iterations when progress is not achieved.

In the next section, we present the new line search SQP algorithm, giving particular attention to the dynamic strategy for updating the penalty parameter. The convergence properties of the algorithm are analyzed in Section 3, and numerical experiments are reported in Section 4.

\section{A Line Search Penalty Method}

Penalty methods attack the general nonlinear programming problem

$$
\begin{aligned}
\operatorname{minimize} & f(x) \\
\text { subject to } & g_{i}(x) \geq 0, \quad i \in \mathcal{I}, \\
& h_{i}(x)=0, \quad i \in \mathcal{E}
\end{aligned}
$$


by performing an unconstrained minimization of the exact penalty function

$$
\phi_{\pi}(x)=f(x)+\pi v(x),
$$

where $v(x)$ is a measure of constraint violation and $\pi$ is the penalty parameter. The penalty approach proposed in this paper is applicable to a variety of line search methods; for concreteness we focus our discussion on sequential quadratic programming.

We use the 1-norm of the constraint violation as the measure of infeasibility for the nonlinear program (2.1). Thus the penalty function is defined by (2.2) with

$$
v(x)=\sum_{i \in \mathcal{I}}\left[g_{i}(x)\right]^{-}+\sum_{i \in \mathcal{E}}\left|h_{i}(x)\right|
$$

where $\left[g_{i}(x)\right]^{-}=\max \left\{-g_{i}(x), 0\right\}$ is the negative part of $g_{i}(x)$.

It is well known (see for example [21]) that when multipliers exist, stationary points of the nonlinear problem (2.1) are also stationary points of the exact penalty function (2.2) for all sufficiently large values of $\pi$. Conversely, and more important from the standpoint of practical penalty methods, any stationary point of the exact penalty function (2.2) that is feasible for problem (2.1) is a stationary point of (2.1).

Exact penalty methods attempt to find stationary points of the nonlinear program (2.1) by minimizing the penalty function (2.2), and use the exogenous penalty parameter $\pi$ as a control to promote feasibility. Two key questions arise: a) How can we find stationary points of the nonsmooth exact penalty function $\phi_{\pi}$, for a fixed value of $\pi$ ? b) How should we update the penalty parameter $\pi$ ?

The first of these issues is well understood [13]. We can search for stationary points of the penalty function by taking steps based on a quadratic model of $\phi_{\pi}$. To define this model we first construct the following piecewise linear model of the measure of constraint violation $v$ at an iterate $x_{k}$ :

$$
m_{k}(d)=\sum_{i \in \mathcal{I}}\left[\nabla g_{i}\left(x_{k}\right)^{T} d+g_{i}\left(x_{k}\right)\right]^{-}+\sum_{i \in \mathcal{E}}\left|\nabla h_{i}\left(x_{k}\right)^{T} d+h_{i}\left(x_{k}\right)\right| .
$$

Next, we define a piecewise quadratic model of $\phi_{\pi}$ at $x_{k}$ as

$$
q_{k}^{\pi}(d)=f\left(x_{k}\right)+\nabla f\left(x_{k}\right)^{T} d+\frac{1}{2} d^{T} W_{k} d+\pi m_{k}(d),
$$

where $W_{k}$ is a symmetric positive definite matrix that approximates the Hessian of the Lagrangian of the nonlinear program (2.1). We compute the search direction $d_{k}$ by solving the problem

$$
\underset{d}{\operatorname{minimize}} q_{k}^{\pi}(d)
$$

In practice, we recast (2.6) as the smooth quadratic optimization program

$$
\begin{array}{cl}
\underset{d, r, s, t}{\operatorname{minimize}} & f\left(x_{k}\right)+\nabla f\left(x_{k}\right)^{T} d+\frac{1}{2} d^{T} W_{k} d+\pi \sum_{i \in \mathcal{E}}\left(r_{i}+s_{i}\right)+\pi \sum_{i \in \mathcal{I}} t_{i} \\
\text { subject to } & \nabla h_{i}\left(x_{k}\right)^{T} d+h_{i}\left(x_{k}\right)=r_{i}-s_{i}, \quad i \in \mathcal{E}, \\
& \nabla g_{i}\left(x_{k}\right)^{T} d+g_{i}\left(x_{k}\right) \geq-t_{i}, \quad i \in \mathcal{I} \\
& r, s, t \geq 0 .
\end{array}
$$


Once the solution $d_{k}$ of problem (2.6) is found, a line search is performed in the direction $d_{k}$ to ensure that sufficient decrease in the exact penalty function $(2.2)$ is achieved at the new iterate.

The positive definiteness assumption on $W_{k}$ is common in line search methods, where $W_{k}$ is obtained through a quasi-Newton update or by adding (if necessary) a multiple of the identity to the Hessian of the Lagrangian of problem (2.1). Note that the quadratic subproblem (2.7) is always feasible, and we show in this paper that the introduction of the surplus variables $r, s, t$, together with the positive definiteness of $W_{k}$ provide a regularization effect to the algorithm.

The second challenge in penalty methods concerns the selection of the penalty parameter. If $\pi$ is too small, the penalty function (2.2) may be unbounded below, and the iterates will diverge unless the value of $\pi$ is corrected in time. If $\pi$ is too large, the efficiency of the penalty approach may be impaired [8]. The goal of this paper is to propose a dynamic strategy for updating the penalty parameter in a class of line search methods for nonlinear programming.

To describe this strategy, we denote the solution of $(2.6)$ by $d_{k}(\pi)$ to stress its dependence on the penalty parameter. At iteration $k$, we first solve (2.6) using the current penalty parameter $\pi_{k}$, to obtain $d_{k}\left(\pi_{k}\right)$. We are content if the linearized constraints are satisfied, i.e., if $m_{k}\left(d_{k}\left(\pi_{k}\right)\right)=0$. In this case, the penalty parameter is not changed and we define the search direction as $d_{k} \triangleq d_{k}\left(\pi_{k}\right)$. Thus, no regularization is needed in this case and the search direction $d_{k}$ coincides with that computed by a classical SQP method (in which $r, s, t$ are always zero).

On the other hand, if $m_{k}\left(d_{k}\left(\pi_{k}\right)\right)>0$, we assess the adequacy of the current penalty parameter by computing the lowest possible violation of the linearized constraints in a neighborhood of the current iterate. This is done by solving the problem

$$
\underset{d}{\operatorname{minimize}} m_{k}(d), \quad \text { subject to }\|d\|_{\infty} \leq \Delta_{k},
$$

where $\Delta_{k}>0$ is given. This problem is equivalent to the linear program

$$
\begin{array}{cl}
\underset{d, r, s, t}{\operatorname{minimize}} & \sum_{i \in \mathcal{E}}\left(r_{i}+s_{i}\right)+\sum_{i \in \mathcal{I}} t_{i} \\
\text { subject to } & \nabla h_{i}\left(x_{k}\right)^{T} d+h_{i}\left(x_{k}\right)=r_{i}-s_{i}, \quad i \in \mathcal{E}, \\
& \nabla g_{i}\left(x_{k}\right)^{T} d+g_{i}\left(x_{k}\right) \geq-t_{i}, \quad i \in \mathcal{I}, \\
& \|d\|_{\infty} \leq \Delta_{k}, \\
& r, s, t \geq 0 .
\end{array}
$$

We denote the solution of this problem by $d_{k}^{\mathrm{LP}}$. A new penalty parameter $\pi_{+} \geq \pi_{k}$ is now determined such that the solution $d_{k}\left(\pi_{+}\right)$of problem (2.6) yields an improvement in linearized feasibility that is commensurate with that obtained by the step $d_{k}^{\mathrm{LP}}$, as measured by the model $m_{k}$. This strategy is specified more precisely in Algorithm I, which is the new line search penalty method. 


\section{Algorithm I: Line Search Penalty SQP Method}

Initial data: $x_{1}, \pi_{1}>0, \rho>0, \epsilon_{1} \in(0,1], \epsilon_{2} \in\left(0, \epsilon_{1}\right), \tau \in(0,1), \eta \in(0,1)$, and $0<\Delta_{\min } \leq \Delta_{1} \leq \Delta_{\max }$.

For $k=1,2, \ldots$

1. Find a search direction $d_{k}\left(\pi_{k}\right)$ by solving the subproblem (2.6) with $\pi=\pi_{k}$. If $d_{k}\left(\pi_{k}\right)=0$ and $v\left(x_{k}\right)=0$, STOP: $x_{k}$ is a KKT point of problem (2.1).

2. If $m_{k}\left(d_{k}\left(\pi_{k}\right)\right)=0$, set $\pi_{+}=\pi_{k}$ and go to Step 6 .

3. Solve the linear programming subproblem $(2.8)$ to get $d_{k}^{\mathrm{LP}}$. If

$$
0<m_{k}(0)=m_{k}\left(d_{k}^{\mathrm{LP}}\right),
$$

STOP: $x_{k}$ is an infeasible stationary point of the penalty function (2.2).

4. Update the penalty parameter:

(a) If $m_{k}\left(d_{k}^{\mathrm{LP}}\right)=0$, find $\pi_{+} \geq \pi_{k}+\rho$ and a corresponding vector $d_{k}\left(\pi_{+}\right)$that solves (2.6), such that

$$
m_{k}\left(d_{k}\left(\pi_{+}\right)\right)=0
$$

(b) Else set $\pi_{+}=\pi_{k}$. If the following inequality does not hold

$$
m_{k}(0)-m_{k}\left(d_{k}\left(\pi_{+}\right)\right) \geq \epsilon_{1}\left[m_{k}(0)-m_{k}\left(d_{k}^{\mathrm{LP}}\right)\right]
$$

then find $\pi_{+} \geq \pi_{k}+\rho$ and a corresponding vector $d_{k}\left(\pi_{+}\right)$, such that $(2.12)$ is satisfied.

5. If the following inequality does not hold

$$
q_{k}^{\pi_{+}}(0)-q_{k}^{\pi_{+}}\left(d_{k}\left(\pi_{+}\right)\right) \geq \epsilon_{2} \pi_{+}\left[m_{k}(0)-m_{k}\left(d_{k}^{\mathrm{LP}}\right)\right],
$$

then increase $\pi_{+}$(by at least $\rho$ ) as necessary, until the solution $d_{k}\left(\pi_{+}\right)$of $(2.6)$ satisfies (2.13).

6. Set $d_{k}=d_{k}\left(\pi_{+}\right)$, and let $0<\alpha_{k} \leq 1$ be the first member of the sequence $\left\{1, \tau, \tau^{2}, \ldots\right\}$ such that

$$
\phi_{\pi_{+}}\left(x_{k}\right)-\phi_{\pi_{+}}\left(x_{k}+\alpha_{k} d_{k}\right) \geq \eta \alpha_{k}\left[q_{k}^{\pi_{+}}(0)-q_{k}^{\pi_{+}}\left(d_{k}\right)\right] .
$$

7. Set $\Delta_{k+1} \in\left[\Delta_{\min }, \Delta_{\max }\right]$.

8. Let $\pi_{k+1}=\pi_{+}$and $x_{k+1}=x_{k}+\alpha_{k} d_{k}$. 
It is worth emphasizing that Algorithm I is a line search method. The global convergence results established in the next section are based on the properties of line search methods together with the regularization effects of the penalty approach. The trust region constraint in (2.8) plays an indirect role, since it only influences the choice of the penalty parameter.

Note that since the Hessian $W_{k}$ in the piecewise quadratic model (2.5) is positive definite, $q_{k}^{\pi}$ is strictly convex. Therefore the solution of problem $(2.6)$, which we denote by $d_{k}(\pi)$, is unique. The model $m(d)$ is convex, but not strictly convex.

The overall design of Algorithm I is based on the following three updating guidelines, which we call the steering rules and are an adaptation of the strategy given in [8] to the line search setting:

1. If it is possible to satisfy the linearized constraints in a neighborhood of the current iterate, we compute such a step. This is achieved by enforcing condition (2.11). In other words, if a classical SQP step exists and is not too long, we would like to use it.

2. If the linearized constraints are locally infeasible, we will be content with taking a step that achieves at least a fraction of the best possible local feasibility improvement. We impose this requirement through condition (2.12). Note that (2.12) could be satisfied with the current penalty parameter $\pi_{k}$, in which case no extra quadratic subproblems (2.6) need to be solved.

3. Not only feasibility but also improvement in the penalty function has to be commensurate with the improvement in feasibility obtained with $d_{k}^{\mathrm{LP}}$. This is guaranteed by condition (2.13). We note that it is not necessary to re-solve problem (2.6) when condition (2.13) is violated because an appropriate value of the penalty parameter is readily computed. First, if $m_{k}(0)-m_{k}\left(d_{k}^{\mathrm{LP}}\right)=0$ then (2.13) is satisfied for any $\pi$ because $d_{k}(\pi)$ is the minimizer of $q_{k}^{\pi}$. Otherwise, $m_{k}(0)>m_{k}\left(d_{k}^{\mathrm{LP}}\right)$, which implies that $m_{k}(0)>0$. Let $\pi_{+}$be the value of the penalty at the beginning of Step 5 of Algorithm I, let $d_{+}=d_{k}\left(\pi_{+}\right)$and define

$$
\hat{\pi}=\frac{\frac{1}{2} d_{+}^{T} W_{k} d_{+}+\nabla f\left(x_{k}\right)^{T} d_{+}}{\left(1-\epsilon_{2}\right) m_{k}(0)-m_{k}\left(d_{+}\right)+\epsilon_{2} m_{k}\left(d_{k}^{\mathrm{LP}}\right)}+\rho .
$$

(Note that (2.11) or (2.12), together with the relations $0<\epsilon_{2}<\epsilon_{1} \leq 1$, imply that the denominator is positive.) Then, by writing $\hat{d}=d(\hat{\pi})$ we have from $(2.15)$ that

$$
\begin{aligned}
\epsilon_{2} \hat{\pi}\left[m_{k}(0)-m_{k}\left(d_{k}^{\mathrm{LP}}\right)\right] & \leq-\nabla f\left(x_{k}\right)^{T} d_{+}-\frac{1}{2} d_{+}^{T} W_{k} d_{+}+\hat{\pi}\left[m_{k}(0)-m_{k}\left(d_{+}\right)\right] \\
& =q_{k}^{\hat{\pi}}(0)-q_{k}^{\hat{\pi}}\left(d_{+}\right) \\
& \leq q_{k}^{\hat{\pi}}(0)-q_{k}^{\hat{\pi}}(\hat{d}),
\end{aligned}
$$

where the last inequality follows from the fact that $\hat{d}$ is the minimizer of $q_{\hat{\pi}}^{k}$. Therefore condition (2.13) is satisfied if the penalty parameter is given by (2.15).

We show in Lemma 3.5-(b) that if the penalty parameter is increased in Step 5 to satisfy (2.13), it will still satisfy conditions (2.11) and (2.12). 
When the penalty parameter is updated in Algorithm I, the main cost is in the repeated solution of the quadratic program (2.6) for different values of $\pi$ in Step 4(b); the linear program (2.8) is solved only once. We expect, however, that warm starts can greatly accelerate the solution of these quadratic programs and that the savings in total iterations will overcome any extra cost incurred in some iterations.

The choice of penalty parameter $\pi_{+}$in Algorithm I ensures that $d_{k}$ is a descent direction for the penalty function (see Lemma 3.5 (c)). Note that in the right-hand side of $(2.14)$ we use the decrease in the piecewise quadratic model $q_{k}^{\pi_{+}}$, instead of the directional derivative of the penalty function. This accounts for possible kinks in the merit function near the current iterate that could be overlooked by a standard Armijo line search and result in jamming.

The constraint $\|d\|_{\infty} \leq \Delta_{k}$ in problem (2.8) is not a trust region in the usual sense, and the value of $\Delta_{k}$ is not critical to the performance of the algorithm. The function $m_{k}(d)$ is always bounded below and the radius $\Delta_{k}$ is used only to ensure that $\left\|d_{k}^{\mathrm{LP}}\right\|$ is of reasonable size in case there is an unbounded ray of minimizers of $m_{k}(d)$. In fact, the radius $\Delta_{k}$ could be kept constant and the convergence properties of Algorithm I would not be affected. In practice, however, it may be advantageous to choose $\Delta_{k}$ based on local information of the problem, as discussed in Section 4.

\section{Convergence analysis}

In this section we study the global convergence properties of Algorithm I. We make the following assumptions about the sequence of iterates $\left\{x_{k}\right\}$ and the matrices $W_{k}$ generated by the algorithm.

\section{Assumptions I.}

A1. The functions $f, g_{i}, i \in \mathcal{I}$, and $h_{i}, i \in \mathcal{E}$, are twice differentiable with bounded derivatives over a bounded convex set that contains the sequence $\left\{x_{k}\right\}$.

A2. The matrices $W_{k}$ are uniformly positive definite and bounded above, i.e., there exist values $0<\mu_{\min }<\mu_{\max }$ such that

$$
\mu_{\min }\|p\|^{2} \leq p^{T} W_{k} p \leq \mu_{\max }\|p\|^{2},
$$

for an $p \in \mathbb{R}^{n}$.

We denote the directional derivative of a function $f$ at $x$ in the direction $p$ by $D f(x ; p)$. A point $x$ is said to be a stationary point of the penalty function if $D \phi_{\pi}(x ; p) \geq 0$ for all directions $p$. A point $\hat{x}$ is called an infeasible stationary point for problem (2.1) if $v(\hat{x})>0$ and $D v(\hat{x} ; p) \geq 0$ for all $p$. We say that problem (2.1) is locally infeasible if there is an infeasible stationary point for it.

The first lemma provides useful relationships between the directional derivatives of the functions $\phi_{\pi}$ and $v$ and their local models, $q^{\pi}$ and $m$. 
Lemma 3.1 Given a point $x_{k}$, the directional derivatives of $v$ and $\phi_{\pi}$ along a vector $p$ satisfy

$$
D v\left(x_{k} ; p\right)=D m_{k}(0 ; p)
$$

and

$$
D \phi_{\pi}\left(x_{k} ; p\right)=D q_{k}^{\pi}(0 ; p)
$$

Proof. Given $x_{k}$ and a vector $d \in \mathbb{R}^{n}$, let us define the sets

$$
\begin{aligned}
& \mathcal{G}_{-}^{k}(d)=\left\{i \in \mathcal{I}: \nabla g_{i}\left(x_{k}\right)^{T} d+g_{i}\left(x_{k}\right)<0\right\}, \\
& \mathcal{G}_{0}^{k}(d)=\left\{i \in \mathcal{I}: \nabla g_{i}\left(x_{k}\right)^{T} d+g_{i}\left(x_{k}\right)=0\right\}, \\
& \mathcal{G}_{+}^{k}(d)=\left\{i \in \mathcal{I}: \nabla g_{i}\left(x_{k}\right)^{T} d+g_{i}\left(x_{k}\right)>0\right\},
\end{aligned}
$$

which determine a partition of $\mathcal{I}$. Similarly, we can define a partition $\mathcal{H}_{-}^{k}(d), \mathcal{H}_{0}^{k}(d)$ and $\mathcal{H}_{+}^{k}(d)$ of $\mathcal{E}$ induced by the value of $\nabla h_{i}\left(x_{k}\right)^{T} d+h_{i}\left(x_{k}\right)$.

The directional derivative of $m_{k}(\cdot)$ at $d$ in the direction $p$ is given by

$$
\begin{aligned}
D m_{k}(d ; p)= & \sum_{i \in \mathcal{G}_{0}^{k}(d)}\left[\nabla g_{i}\left(x_{k}\right)^{T} p\right]^{-}-\sum_{i \in \mathcal{G}_{-}^{k}(d)} \nabla g_{i}\left(x_{k}\right)^{T} p \\
& +\sum_{i \in \mathcal{H}_{+}^{k}(d)} \nabla h_{i}\left(x_{k}\right)^{T} p+\sum_{i \in \mathcal{H}_{0}^{k}(d)}\left|\nabla h_{i}\left(x_{k}\right)^{T} p\right|-\sum_{i \in \mathcal{H}_{-}^{k}(d)} \nabla h_{i}\left(x_{k}\right)^{T} p .
\end{aligned}
$$

On the other hand, we have that

$$
\begin{aligned}
D v\left(x_{k} ; p\right)= & \sum_{i \in \mathcal{G}_{0}^{k}(0)}\left[\nabla g_{i}\left(x_{k}\right)^{T} p\right]^{-}-\sum_{i \in \mathcal{G}_{-}^{k}(0)} \nabla g_{i}\left(x_{k}\right)^{T} p \\
& +\sum_{i \in \mathcal{H}_{+}^{k}(0)} \nabla h_{i}\left(x_{k}\right)^{T} p+\sum_{i \in \mathcal{H}_{0}^{k}(0)}\left|\nabla h_{i}\left(x_{k}\right)^{T} p\right|-\sum_{i \in \mathcal{H}_{-}^{k}(0)} \nabla h_{i}\left(x_{k}\right)^{T} p .
\end{aligned}
$$

The equality (3.2) follows by comparing this expression with (3.5).

Given a direction $p$, we have that $D \phi_{\pi}\left(x_{k} ; p\right)=\nabla f\left(x_{k}\right)^{T} p+D v\left(x_{k} ; p\right)$. Also, for any $d$ we have that $D q_{k}^{\pi}(d ; p)=\left(\nabla f\left(x_{k}\right)+W_{k} d\right)^{T} p+D m_{k}(d ; p)$. By evaluating $D q_{k}^{\pi}(d ; p)$ at $d=0$ and using (3.2) we obtain (3.3).

The next result is well known (see e.g. [4, 21]). For a given point $x_{*}$, we define the model $(2.5)$ at $x_{*}$ by $q_{*}^{\pi}$, and denote its solution by $d_{*}(\pi)$. Similarly, $m_{*}$ denotes the model (2.4) at $x_{*}$.

Theorem 3.2 The following three statements are true:

(a) $x_{*}$ is a stationary point of the penalty function $\phi_{\pi}(x)$ if and only if $d_{*}(\pi)=0$ solves problem (2.6).

(b) If $x_{*}$ is a stationary point of $\phi_{\pi}(x)$ and $v\left(x_{*}\right)=0$, then $x_{*}$ is a KKT point of (2.1).

(c) $x_{*}$ is a stationary point of the infeasibility measure $v(x)$ if and only if, for any $\Delta>0$, any solution $d^{L P}$ of the linear feasibility problem (2.8) satisfies

$$
m_{*}\left(d^{L P}\right)=m_{*}(0)
$$


Proof. (a) By definition, $d_{*}(\pi)=0$ is the minimizer of $q_{*}^{\pi}(d)$ if and only if $D q_{*}^{\pi}(0 ; p) \geq 0$ for any direction $p$. The result follows from (3.3). (b) See [26, Theorem 17.4].

(c) Given $\Delta>0$, let $d^{\mathrm{LP}}$ be a solution of (2.8). Since $d=0$ obviously satisfies $\|d\|_{\infty} \leq \Delta$, we have that $m_{*}\left(d^{\mathrm{LP}}\right) \leq m_{*}(0)$. Also, from (3.2) we have that $x_{*}$ is stationary for $v(x)$ if and only if 0 is stationary for $m_{*}(d)$, which holds (by convexity of $m_{*}$ ) if and only if 0 is an unconstrained global minimizer of $m_{*}(d)$. Therefore, $m_{*}(0) \leq m_{*}(d)$ for any $d$, and in particular for $d=d^{\mathrm{LP}}$. We conclude that (3.6) holds.

This theorem justifies the stopping tests in Algorithm I. If Algorithm I stops at Step 1, Theorem 3.2 (a) and (b) imply that the current iterate $x_{k}$ is a KKT point of the nonlinear program (2.1). If the algorithm stops at Step 3, then Theorem 3.2 (c) and $v\left(x_{k}\right)>0$ imply that $x_{k}$ is an infeasible stationary point. If neither stop test is satisfied, we need to show that that Algorithm I will generate a new iterate $x_{k+1}$ and that it is always possible to meet the requirements in Steps 4 and 6. This is done in Lemma 3.5; first we need to establish two auxiliary results.

Lemma 3.3 Suppose that Assumptions I hold. At any given iterate $x_{k}$, and for all $\pi>0$, the minimizers $d_{k}(\pi)$ of $q_{k}^{\pi}(d)$ are contained in a compact ball

$$
\mathcal{B}^{k}=\left\{d:\|d\| \leq r^{k}\right\} \quad \text { with } \quad r^{k}=\kappa_{1}+\kappa_{2}\left\|\bar{d}_{k}\right\|,
$$

for some global constants $\kappa_{1}$ and $\kappa_{2}$ and where $\bar{d}_{k}$ denotes the minimum norm minimizer of $m_{k}(d)$.

Proof. Let $\bar{d}_{k}$ be the minimum norm minimizer of $m_{k}(d)$; it is well defined because $m_{k}(d)$ is a piece-wise linear convex function that is bounded below. If $\|d\|$ is large enough that $\mu_{\text {min }}\|d\| \geq 8\left\|\nabla f\left(x_{k}\right)\right\|$ and $\mu_{\min }\|d\|^{2} \geq 2 \mu_{\max }\|\bar{d}\|^{2}$, then by $(3.1)$

$$
\begin{aligned}
-\nabla f\left(x_{k}\right)^{T} d+\nabla f\left(x_{k}\right)^{T} \bar{d}+\frac{1}{2} \bar{d}^{T} W_{k} \bar{d} & \leq\left\|\nabla f_{k}\right\|\|d\|+\left\|\nabla f_{k}\right\|\|\bar{d}\|+\frac{\mu_{\max }}{2}\|\bar{d}\|^{2} \\
& \leq \frac{\mu_{\min }}{8}\|d\|^{2}+\frac{\mu_{\min }}{8}\left[\frac{\mu_{\min }}{2 \mu_{\max }}\right]^{\frac{1}{2}}\|d\|^{2}+\frac{\mu_{\min }}{4}\|d\|^{2} \\
& <\frac{\mu_{\min }}{2}\|d\|^{2} \\
& \leq \frac{1}{2} d^{T} W d,
\end{aligned}
$$

and therefore

$$
f\left(x_{k}\right)+\nabla f\left(x_{k}\right)^{T} d+\frac{1}{2} d^{T} W_{k} d>f\left(x_{k}\right)+\nabla f\left(x_{k}\right)^{T} \bar{d}+\frac{1}{2} \bar{d}^{T} W_{k} \bar{d} .
$$

Thus, for all

$$
\|d\|>\max \left\{8\left\|\nabla f\left(x_{k}\right)\right\| / \mu_{\min }, \sqrt{2 \mu_{\max } / \mu_{\min }}\|\bar{d}\|\right\}
$$

and all $\pi \geq 0$ we have

$$
\begin{aligned}
q_{k}^{\pi}(d) & =f\left(x_{k}\right)+\nabla f\left(x_{k}\right)^{T} d+\frac{1}{2} d^{T} W_{k} d+\pi m_{k}(d) \\
& >f\left(x_{k}\right)+\nabla f\left(x_{k}\right)^{T} \bar{d}+\frac{1}{2} \bar{d}^{T} W_{k} \bar{d}+\pi m_{k}(\bar{d})=q_{k}^{\pi}(\bar{d}),
\end{aligned}
$$


since $\bar{d}$ is a minimizer of $m_{k}$. Therefore, no minimizer $d_{k}(\pi)$ can be larger in norm than the right hand side of (3.8). To establish (3.7), we let $\tilde{\kappa}_{1}$ be an upper bound for $\left\|\nabla f_{k}\right\|$, define $\kappa_{1}=8 \tilde{\kappa}_{1} / \mu_{\min }$ and $\kappa_{2}=\sqrt{2 \mu_{\max } / \mu_{\min }}$.

The following result shows that by choosing $\pi$ sufficiently large, the direction $d(\pi)$ can attain any achievable level of linear feasibility.

Lemma 3.4 At any iterate $x_{k}$, for all all $\pi$ sufficiently large the minimizer $d_{k}(\pi)$ of $q_{k}^{\pi}$, also minimizes $m_{k}(d)$.

Proof. It is clear from (2.4) that the piecewise linear function $m_{k}(d)$ may be expressed as

$$
m_{k}(d)=\max _{j \in \mathcal{M}}\left\{a_{j}^{T} d+\beta_{j}\right\},
$$

where $\mathcal{M}$ is a finite index set, the vectors $a_{j}$ are in $\mathbb{R}^{n}$ and the $\beta_{j}$ are scalars. It follows (see e.g. [28, p.66]) that for any $d \in \mathbb{R}^{n}$ the subdifferential $\partial m_{k}(d)$ is the convex hull of the active support functions at $d$, i.e.

$$
\partial m_{k}(d)=\operatorname{conv}\left\{a_{j} \mid j \in \mathcal{M} \text { and } a_{j}^{T} d+\beta_{j}=m_{k}(d)\right\} .
$$

Since a vector $d$ minimizes the convex function $m_{k}$ if and only if $\partial m_{k}(d)$ contains 0 , then if $d$ is not a minimizer of $m_{k}$ it follows that $\partial m_{k}(d)$ is a closed convex set not containing 0 , which implies that

$$
\sigma(d) \triangleq \min \left\{\|a\| \mid a \in \partial m_{k}(d)\right\}>0
$$

Now since $\partial m_{k}(d)$ is defined by the active set $\left\{j \in \mathcal{M} \mid a_{j}^{T} d+\beta_{j}=m_{k}(d)\right\}$ and only a finite number of possible sets $\partial m_{k}(d)$ exist as $d$ ranges over $\mathbb{R}^{n}$, the function $\sigma(d)$ takes on only a finite set of values. Therefore

$$
\sigma_{k} \triangleq \min _{d \in \mathbb{R}^{n}}\left\{\sigma(d) \mid 0 \notin \partial m_{k}(d)\right\}>0 .
$$

Now by Lemma 3.3 , there is a compact ball $\mathcal{B}^{k}$ containing the minimizers of $q_{k}^{\pi}$, for all $\pi$. Therefore, for all such minimizers $d_{k}(\pi)$ we have $\left\|\nabla f\left(x_{k}\right)+W_{k} d_{k}(\pi)\right\| \leq \beta_{k}$, for some constant $\beta_{k}$. Consider some $\tilde{\pi}>\beta_{k} / \sigma_{k}$ and the minimizer $d_{k}(\tilde{\pi})$ of $q_{k}^{\tilde{\pi}}$. Any vector $g \in \partial q_{k}^{\tilde{\pi}}\left(d_{k}(\tilde{\pi})\right)$ may be expressed as

$$
g=\nabla f\left(x_{k}\right)+W_{k} d_{k}(\tilde{\pi})+\tilde{\pi} a \quad \text { for some } \quad a \in \partial m_{k}\left(d_{k}(\tilde{\pi})\right)
$$

If $d_{k}(\tilde{\pi})$ does not minimize $m_{k}$, it follows from (3.9) that

$$
\|g\| \geq \tilde{\pi}\|a\|-\left\|\nabla f\left(x_{k}\right)+W_{k} d_{k}(\pi)\right\| \geq \tilde{\pi} \sigma_{k}-\beta_{k}>0
$$

This means $0 \notin \partial q_{k}^{\tilde{\pi}}$ contradicting the fact that $d_{k}(\tilde{\pi})$ is a minimizer of $q_{k}^{\tilde{\pi}}$. Therefore $d_{k}(\tilde{\pi})$ must minimize $m_{k}$.

For the remainder of the analysis, it is useful to define the model

$$
q_{k}^{f}(d)=f_{k}+\nabla f_{k}^{T} d+\frac{1}{2} d^{T} W_{k} d
$$

so that

$$
q_{k}^{\pi_{k}}(d)=q_{k}^{f}(d)+\pi_{k} m_{k}(d)
$$

We now prove that Algorithm I is well defined. 
Lemma 3.5 Suppose that $x_{k}$ is neither a KKT point of nonlinear program (2.1) nor a stationary point of the infeasibility measure $v(x)$. Then,

a) If Algorithm I executes Step 4, it is always possible to find a value $\pi_{+}$and a corresponding vector $d_{k}\left(\pi_{+}\right)$such that condition $(2.11)$ holds if $m_{k}\left(d^{L P}\right)=0$, or condition $(2.12)$ holds if $m_{k}\left(d^{L P}\right)>0$.

b) If Algorithm I executes Step 5, it is always possible to find a value $\pi_{+}$and a corresponding vector $d_{k}\left(\pi_{+}\right)$such that condition (2.13) holds.

c) At Step $6, d_{k}$ is a descent direction for $\phi_{\pi_{+}}(x)$ at $x_{k}$. Therefore, there exists $\alpha_{k}$ such that condition (2.14) is satisfied.

Proof. (a) By Lemma 3.4, for $\pi_{+}$sufficient large $d_{k}\left(\pi_{+}\right)$is a minimizer of $m_{k}(d)$ and hence $m_{k}\left(d_{k}\left(\pi_{+}\right)\right) \leq m_{k}\left(d_{k}^{\mathrm{LP}}\right)$; this implies (2.11). Moreover, since $\epsilon_{1} \leq 1$, we have that

$$
m_{k}(0)-m_{k}\left(d_{k}\left(\pi_{+}\right)\right) \geq m_{k}(0)-m_{k}\left(d_{k}^{\mathrm{LP}}\right) \geq \epsilon_{1}\left[m_{k}(0)-m_{k}\left(d_{k}^{\mathrm{LP}}\right)\right],
$$

so that (2.12) is satisfied.

b) We have already shown in Section 2 that (2.13) is satisfied if the penalty parameter is chosen by (2.15). We now show that if the penalty parameter is increased in Step 5, this new value of $\pi$ still satisfies (2.11) and (2.12).

Let $\pi_{2}>\pi_{1}$. Then by (3.11) and the fact that $d_{k}\left(\pi_{k}\right)$ is the minimizer of $q_{\pi}^{k}$, we have

$$
\begin{aligned}
q_{k}^{f}\left(d_{k}\left(\pi_{1}\right)\right)+\pi_{2} m_{k}\left(d_{k}\left(\pi_{1}\right)\right) & \geq q_{k}^{f}\left(d_{k}\left(\pi_{2}\right)\right)+\pi_{2} m_{k}\left(d_{k}\left(\pi_{2}\right)\right) \\
q_{k}^{f}\left(d_{k}\left(\pi_{1}\right)\right)+\pi_{1} m_{k}\left(d_{k}\left(\pi_{1}\right)\right) & \leq q_{k}^{f}\left(d_{k}\left(\pi_{2}\right)\right)+\pi_{1} m_{k}\left(d_{k}\left(\pi_{2}\right)\right) .
\end{aligned}
$$

Hence

$$
\left(\pi_{2}-\pi_{1}\right) m_{k}\left(d_{k}\left(\pi_{1}\right)\right) \geq\left(\pi_{2}-\pi_{1}\right) m_{k}\left(d_{k}\left(\pi_{2}\right)\right)
$$

which implies that $m_{k}\left(d_{k}\left(\pi_{1}\right)\right) \geq m_{k}\left(d_{k}\left(\pi_{2}\right)\right)$. We conclude that $m_{k}\left(d_{k}(\pi)\right)$ cannot increase as $\pi$ is increased.

(c) If $x_{k}$ is neither stationary for $\phi_{\pi_{k}}$ nor for $v(x)$, then at Step 6 we must have $d_{k} \neq$ 0 . This is a consequence of the logic of Algorithm I and of Theorem 3.2, parts (a) and (c). Recall that $d_{k}$ is defined to be the minimizer of $q_{k}^{\pi_{+}}$and hence $q_{k}^{\pi_{+}}\left(d_{k}\right) \leq q_{k}^{\pi_{+}}(0)$. Furthermore, since $q_{k}^{\pi_{+}}(d)$ is strictly convex and $d_{k} \neq 0$, we have that $q_{k}^{\pi_{+}}\left(d_{k}\right)<q_{k}^{\pi_{+}}(0)$ and thus $d_{k}$ is a descent direction for $q_{k}^{\pi_{+}}$at 0 . By (3.3), we have

$$
D \phi_{\pi_{+}}\left(x_{k} ; d_{k}\right)=D q_{k}^{\pi_{+}}\left(0 ; d_{k}\right)<0,
$$

and therefore $d_{k}$ is also a descent direction for $\phi_{\pi_{+}}(x)$ at $x_{k}$. Since the constant $\eta$ is chosen in $(0,1)$, it follows that $\phi_{\pi_{+}}\left(x_{k}+\alpha d_{k}\right)<\phi_{\pi_{+}}\left(x_{k}\right)+\alpha \eta D \phi_{\pi_{+}}\left(x_{k} ; d_{k}\right)$ for all sufficiently small $\alpha>0$, or

$$
\phi_{\pi_{+}}\left(x_{k}\right)-\phi_{\pi_{+}}\left(x_{k}+\alpha d_{k}\right)>-\alpha \eta D \phi_{\pi_{+}}\left(x_{k} ; d_{k}\right) .
$$

From (3.3) and the convexity of $q_{\pi_{+}}^{k}(d)$, we have that

$$
-\alpha \eta D \phi_{\pi_{+}}\left(x_{k} ; d_{k}\right)=-\alpha \eta D q_{\pi_{+}}^{k}\left(0 ; d_{k}\right) \geq \alpha \eta\left[q_{k}^{\pi_{+}}(0)-q_{k}^{\pi_{+}}\left(d_{k}\right)\right] .
$$


We conclude that there always exists a sufficiently small steplength $\alpha_{k}$ that satisfies condition (2.14).

We now establish the first convergence result. It gives conditions under which Algorithm I identifies stationary points of the penalty function.

Theorem 3.6 Suppose that Algorithm I generates an infinite sequence of iterates $\left\{x_{k}\right\}$ and that Assumptions I hold. Suppose also that $\left\{\pi_{k}\right\}$ is bounded, so that $\pi_{k}=\bar{\pi}$ for all $k$ large. Then any accumulation point $x_{*}$ of $\left\{x_{k}\right\}$ is a stationary point of the penalty function $\phi_{\bar{\pi}}(x)$.

Proof. Note that, whenever $\pi$ is increased in Algorithm I, it is increased by at least $\rho>0$. Therefore, if $\left\{\pi_{k}\right\}$ is bounded, there is a value $\bar{\pi}$ such that $\pi_{k}=\bar{\pi}$ for all sufficiently large $k$.

Let $x_{*}$ be a limit point of $\left\{x_{k}\right\}$, which exists because Assumption A1 states that $\left\{x_{k}\right\}$ is bounded. Let $\mathcal{K}$ be an infinite subset of indices such that $\left\{x_{k}\right\}_{k \in \mathcal{K}} \rightarrow x_{*}$. The sequence of matrices $\left\{W_{k}\right\}$ is also bounded, by Assumption A2. We restrict $\mathcal{K}$ if necessary so that $\left\{W_{k}\right\}_{k \in \mathcal{K}} \rightarrow W_{*}$, where $W_{*}$ is a limit point of $\left\{W_{k}\right\}$. Then, from the continuity of the functions $f, g_{i}, h_{i}$ and their gradients, and from the definition (2.5), we have that the sequence of models $q_{k}^{\bar{\pi}}, k \in \mathcal{K}$ converges (pointwise) to a function $q_{*}^{\bar{\pi}}$.

Each of the functions $q_{k}^{\bar{\pi}}$, as well as the limiting function $q_{*}^{\bar{\pi}}$, are strictly convex and have a unique minimizer. We want to prove that the minimizer of $q_{*}^{\bar{\pi}}(d)$ is $d_{*}=0$, for then Theorem 3.2 (a) implies that $x_{*}$ is a stationary point of $\phi_{\bar{\pi}}$.

We proceed by contradiction. Assume that $d_{*} \neq 0$, or equivalently, that $q_{*}^{\bar{\pi}}(0)-q_{*}^{\bar{\pi}}\left(d_{*}\right)>$ 0 . From the pointwise convergence of the functions $q_{k}^{\bar{\pi}}$, we know that there exists $\epsilon>0$ such that

$$
q_{k}^{\bar{\pi}}(0)-q_{k}^{\bar{\pi}}\left(d_{*}\right) \rightarrow q_{*}^{\bar{\pi}}(0)-q_{*}^{\bar{\pi}}\left(d_{*}\right)=2 \epsilon>0 .
$$

Therefore, there is a number $k_{0}$ such that for all $k \geq k_{0}$, with $k \in \mathcal{K}$, we have that $\pi_{k}=\bar{\pi}$ and

$$
q_{k}^{\bar{\pi}}(0)-q_{k}^{\bar{\pi}}\left(d_{k}\right) \geq q_{k}^{\bar{\pi}}(0)-q_{k}^{\bar{\pi}}\left(d_{*}\right) \geq \epsilon .
$$

It is not difficult to show (see e.g. Lemma 3.4 in [6]) that for any $\alpha \in[0,1]$,

$$
\left|\phi_{\bar{\pi}}\left(x_{k}+\alpha d_{k}\right)-q_{k}^{\bar{\pi}}\left(\alpha d_{k}\right)\right| \leq c_{1}\left\|\alpha d_{k}\right\|^{2},
$$

for some positive constant $c_{1}$. Recalling that $q_{k}^{\bar{\pi}}$ is a convex function, noting that $\phi_{\bar{\pi}}\left(x_{k}\right)=$ $q_{k}^{\bar{\pi}}(0)$, using (3.14) and (3.15), and assuming that $\alpha_{k} \leq(1-\eta) \epsilon /\left(c_{1}\left\|d_{k}\right\|^{2}\right)$, we obtain

$$
\begin{aligned}
\phi_{\bar{\pi}}\left(x_{k}\right)-\phi_{\bar{\pi}}\left(x_{k}+\alpha_{k} d_{k}\right) & \geq\left[q_{k}^{\bar{\pi}}(0)-q_{k}^{\bar{\pi}}\left(\alpha_{k} d_{k}\right)\right]-\phi_{\bar{\pi}}\left(x_{k}+\alpha_{k} d_{k}\right)+q_{k}^{\bar{\pi}}\left(\alpha_{k} d_{k}\right) \\
& \geq \alpha_{k}\left[q_{k}^{\bar{\pi}}(0)-q_{k}^{\bar{\pi}}\left(d_{k}\right)\right]+c_{1} \alpha_{k}^{2}\left\|d_{k}\right\|^{2} \\
& =\eta \alpha_{k}\left[q_{k}^{\bar{\pi}}(0)-q_{k}^{\bar{\pi}}\left(d_{k}\right)\right]+(1-\eta) \alpha_{k}\left[q_{k}^{\bar{\pi}}(0)-q_{k}^{\bar{\pi}}\left(d_{k}\right)\right]-c_{1} \alpha_{k}^{2}\left\|d_{k}\right\|^{2} \\
& \geq \eta \alpha_{k}\left[q_{k}^{\bar{\pi}}(0)-q_{k}^{\bar{\pi}}\left(d_{k}\right)\right]+(1-\eta) \alpha_{k} \epsilon-c_{1} \alpha_{k}^{2}\left\|d_{k}\right\|^{2} \\
& \geq \eta \alpha_{k}\left[q_{k}^{\bar{\pi}}(0)-q_{k}^{\bar{\pi}}\left(d_{k}\right)\right] .
\end{aligned}
$$


Thus, for such $\alpha_{k}$ the sufficient decrease condition (2.14) is satisfied, which implies that Step 6 of Algorithm I will always select $\alpha_{k}$ satisfying

$$
\alpha_{k} \geq \min \left\{\tau(1-\eta) \epsilon /\left(c_{1}\left\|d_{k}\right\|^{2}\right), 1\right\}
$$

Now we argue that optimality of $d_{k}$ implies it satisfies the bound

$$
\left\|d_{k}\right\| \leq \frac{2\left\|\nabla f_{k}\right\|}{\mu_{\min }}+\sqrt{\frac{2 \bar{\pi} m_{k}(0)}{\mu_{\min }}} .
$$

This is clear since if (3.17) is violated,

$$
\begin{aligned}
q_{k}^{\bar{\pi}}(d) & =f\left(x_{k}\right)+\nabla f_{k}^{T} d_{k}+\frac{1}{2} d^{T} W_{k} d+\bar{\pi} m_{k}(d) \\
& \geq f\left(x_{k}\right)-\left\|\nabla f_{k}\right\|\left\|d_{k}\right\|+\frac{1}{2} \mu_{\min }\|d\|^{2} \\
& >f\left(x_{k}\right)+\bar{\pi} m_{k}(0) \\
& =q_{k}^{\bar{\pi}}(0),
\end{aligned}
$$

which would mean $d_{k}$ does not minimize $q_{k}^{\bar{\pi}}$.

Together with (3.16), the bound (3.17) implies there is a constant $c_{2}>0$ such that $\alpha_{k}>c_{2}$ for all $k$. Now, using this bound on $\alpha_{k}$ together with (3.14), it follows that Step 6 of Algorithm I guarantees that

$$
\begin{aligned}
\phi_{\bar{\pi}}\left(x_{k}\right)-\phi_{\bar{\pi}}\left(x_{k}+\alpha_{k} d_{k}\right) & \geq \eta \alpha_{k}\left[q_{k}^{\bar{\pi}}(0)-q_{k}^{\bar{\pi}}\left(d_{k}\right)\right] \\
& \geq \eta c_{2} \epsilon
\end{aligned}
$$

This relation implies that $\phi_{\bar{\pi}}\left(x_{k}\right) \rightarrow \infty$, which contradicts Assumption A1. This implies that the hypothesis $q_{*}^{\bar{\pi}}(0)-q_{*}^{\bar{\pi}}\left(d_{*}\right)>0$ is false, and therefore that $x_{*}$ is a stationary point of $\phi_{\bar{\pi}}$.

Now that we have established that, when the penalty parameter is bounded the algorithm will locate a stationary point of $\phi$, the next result shows that such s stationary point is either a KKT point of the nonlinear program (2.1) or a stationary point of the infeasibility measure.

Theorem 3.7 Suppose that Algorithm I generates an infinite sequence of iterates $\left\{x_{k}\right\}$, that Assumptions I hold, and that $\left\{\pi_{k}\right\}$ is bounded. Let $x_{*}$ be any accumulation point of $\left\{x_{k}\right\}$. Then either: (a) $v\left(x_{*}\right)=0$ and $x_{*}$ is a KKT point of $(2.1)$; or (b) $v\left(x_{*}\right)>0$ and $x_{*}$ is a stationary point of $v(x)$.

Proof. Let $x_{*}$ be a limit point of the sequence $\left\{x_{k}\right\}$. Since $\pi_{k}$ is bounded there is a scalar $\bar{\pi}$ such that $\pi_{k}=\bar{\pi}$ for all large $k$. From Theorem 3.6 we have that $x_{*}$ is a stationary point of $\phi_{\bar{\pi}}(x)$.

(a) If $v\left(x_{*}\right)=0$, then by Theorem $3.2(\mathrm{~b}), x_{*}$ is a KKT point of problem (2.1).

(b) In the case when $v\left(x_{*}\right)>0$ we want to show that (3.6) holds for some $\Delta>0$. Let $\mathcal{K}$ be an infinite subset of indices for which $x_{k} \rightarrow x_{*}$ for $k \in \mathcal{K}$. Let $W_{*}$ be a limiting 
matrix of the sequence $\left\{W_{k}\right\}_{k \in \mathcal{K}}$, and let $q_{*}^{\bar{\pi}}(d)$ be the corresponding piecewise quadratic model. Restricting $\mathcal{K}$ further, if necessary, we obtain pointwise convergence of the models, i.e., $q_{k}^{\bar{\pi}}(d) \rightarrow q_{*}^{\bar{\pi}}(d)$ for $k \in \mathcal{K}$. As before, let $d_{k}$ denote the minimizer of $q_{k}^{\bar{\pi}}(d)$.

Since $x_{*}$ is a stationary point of $\phi_{\bar{\pi}}(x)$, by Theorem 3.2 (a) we have that the minimizer of $q_{*}^{\bar{\pi}}(d)$ is $d_{*}=0$. From the pointwise convergence of the models, it follows that $d_{k} \rightarrow 0$, which in turn implies that

$$
q_{k}^{\bar{\pi}}(0)-q_{k}^{\bar{\pi}}\left(d_{k}\right) \rightarrow 0 .
$$

This limit together with (2.13) imply that $m_{k}(0)-m_{k}\left(d_{k}^{\mathrm{LP}}\right) \rightarrow 0$ for $k \in \mathcal{K}$.

We also have pointwise convergence to a limiting piecewise linear model, i.e., $m_{k}(d) \rightarrow$ $m_{*}(d)$, and hence

$$
\begin{aligned}
0 & =\lim _{k \rightarrow \infty, k \in \mathcal{K}}\left[m_{k}(0)-m_{k}\left(d_{k}^{\mathrm{LP}}\right)\right] \\
& =\lim _{k \rightarrow \infty, k \in \mathcal{K}}\left[m_{k}(0)-\min \|d\|_{\infty} \leq \Delta_{k} m_{k}(d)\right] \\
& \geq \lim _{k \rightarrow \infty, k \in \mathcal{K}}\left[m_{k}(0)-\min \|d\|_{\infty} \leq \Delta_{\min } m_{k}(d)\right],
\end{aligned}
$$

where the last inequality follows from the fact that Algorithm I requires that $\Delta_{k} \geq \Delta_{\min }>0$. Therefore, $0<v\left(x_{*}\right)=m_{*}(0)=m_{*}\left(d^{\mathrm{LP}}\left(\Delta_{\min }\right)\right)$ and by Theorem 3.2 (c) we conclude that $x_{*}$ is a stationary point of infeasibility for $v(x)$.

Now we consider the behavior of the algorithm when the penalty parameter increases without bound. The first result focuses on the development of the penalty parameter in a vicinity of an infeasible stationary point.

Lemma 3.8 Suppose that Algorithm I generates a sequence $\left\{x_{k}\right\}$ that satisfies Assumptions I. Let $x_{*}$ be a cluster point of this sequence such that $v\left(x_{*}\right)>0$, and suppose that $m_{*}(0)-m_{*}\left(d_{k}^{L P}\right)>0$. Then, along any subsequence $\left\{x_{k}\right\}_{k \in \mathcal{K}}$ that converges to $x_{*}$ the penalty parameter is updated only a finite number of times.

Proof. We will show that for any subsequence that converges to such a point $x_{*}$, Step 4(a) cannot be executed infinitely often, and that for $x_{k}$ sufficiently close to $x_{*},(2.12)$ and (2.13) are satisfied for sufficiently large $\pi$. This will prove the result because the penalty parameter is only increased in Steps 4 and 5 of Algorithm I.

As a preliminary observation note that, since we assume

$$
m_{*}(0)-m_{*}\left(d_{k}^{\mathrm{LP}}\right)>0,
$$

there exist constants $r>0$ and $\zeta>0$ such that

$$
m_{k}(0)-m_{k}\left(d_{k}^{\mathrm{LP}}\right)>\zeta, \text { for all } x_{k} \in \mathcal{B}^{*} \triangleq\left\{x:\left\|x_{k}-x_{*}\right\|<r\right\} .
$$

Now we study the situations in which the penalty parameter is increased in Algorithm I. This increase can happen in Steps 4(a), 4(b) or 5 of Algorithm I, and we study each case separately. 
Case (i) Consider an iterate $x_{k}$ where Step 4(a) is executed. By (2.13) and (3.25), for any such $k$

$$
q_{k}^{\pi_{+}}(0)-q_{k}^{\pi_{+}}\left(d_{k}\left(\pi_{+}\right)\right) \geq \epsilon_{2} \pi_{+} \zeta .
$$

Now, by the Lipschitz continuity assumptions in A1, one can show [7, Lemma 3.4] that there is a constant $c_{1}$ such that for any $x_{k}$ and any $\pi$

$$
\left|\phi_{\pi}\left(x_{k}+\alpha d_{k}\right)-q_{k}^{\pi}\left(\alpha d_{k}\right)\right| \leq c_{1}(1+\pi)\left\|\alpha d_{k}\right\|^{2} .
$$

Let us consider the sufficient decrease condition (2.14). From the equality $\phi_{\pi_{+}}\left(x_{k}\right)=q_{k}^{\pi_{+}}(0)$, the convexity of $q_{k}^{\pi_{+}},(3.27)$ and (3.26), we have, for $x_{k} \in \mathcal{B}^{*}$,

$$
\begin{aligned}
\phi_{\pi_{+}}\left(x_{k}\right)-\phi_{\pi_{+}}\left(x_{k}+\alpha d_{k}\right)= & \eta \alpha\left[q_{k}^{\pi_{+}}(0)-q_{k}^{\pi_{+}}\left(d_{k}\right)\right] \\
= & {\left[q_{k}^{\pi_{+}}(0)-q_{k}^{\pi_{+}}\left(\alpha d_{k}\right)\right]-\left[\phi_{\pi_{+}}\left(x_{k}+\alpha d_{k}\right)-q_{k}^{\pi_{+}}\left(\alpha d_{k}\right)\right] } \\
& -\eta \alpha\left[q_{k}^{\pi_{+}}(0)-q_{k}^{\pi_{+}}\left(d_{k}\right)\right] \\
\geq & \alpha\left[q_{k}^{\pi_{+}}(0)-q_{k}^{\pi_{+}}\left(d_{k}\right)\right]-c_{1}\left(1+\pi_{+}\right)\left\|\alpha d_{k}\right\|^{2}-\eta \alpha\left[q_{k}^{\pi_{+}}(0)-q_{k}^{\pi_{+}}\left(d_{k}\right)\right] \\
\geq & \alpha(1-\eta)\left[q_{k}^{\pi_{+}}(0)-q_{k}^{\pi_{+}}\left(d_{k}\right)\right]-c_{1}\left(1+\pi_{+}\right)\left\|\alpha d_{k}\right\|^{2} \\
\geq & \alpha(1-\eta) \epsilon_{2} \pi_{+} \zeta-c_{1}\left(1+\pi_{+}\right) \alpha^{2}\left\|d_{k}\right\|^{2} .
\end{aligned}
$$

The right hand side is nonnegative if $\alpha \leq(1-\eta) \epsilon_{2} \pi_{+} \zeta / c_{1}\left(1+\pi_{+}\right)\left\|d_{k}\right\|^{2}$. Therefore Step 6 of Algorithm I will always choose

$$
\alpha_{k} \geq \min \left\{1, \tau(1-\eta) \epsilon_{2} \pi_{+} \zeta / c_{1}\left(1+\pi_{+}\right)\left\|d_{k}\right\|^{2}\right\}
$$

where $\tau$ is the contraction factor used in Step 6 of the algorithm.

Since when Step $4(\mathrm{a})$ is executed $m_{k}\left(d_{k}^{\mathrm{LP}}\right)=0$, we have that $d_{k}^{\mathrm{LP}}$ is an unconstrained minimizer of $m_{k}$ and is thus no smaller in norm than the minimum norm minimizer $\bar{d}_{k}$ mentioned in Lemma 3.3. By applying Lemma 3.3, we obtain the bound $\left\|d_{k}\right\| \leq \kappa_{1}+$ $\kappa_{2}\left\|d_{k}^{\mathrm{LP}}\right\| \leq \kappa_{1}+\kappa_{2} \Delta_{\max }$, since $\left\|d_{k}^{\mathrm{LP}}\right\| \leq \Delta_{\max }$; see Step 7. Using this bound in (3.28) gives

$$
\alpha_{k} \geq \min \left\{1, \frac{(1-\eta) \tau \epsilon_{2} \pi_{+} \zeta}{c_{1}\left(1+\pi_{+}\right)\left(\kappa_{1}+\kappa_{2} \Delta_{\max }\right)^{2}}\right\} \geq c_{2}
$$

for some constant $c_{2}>0$. This bound, together with (2.14) and (3.26) implies that there is a constant $c_{3}>0$ such that for any $x_{k} \in \mathcal{B}^{*}$,

$$
\phi_{\pi_{k+1}}\left(x_{k+1}\right) \leq \phi_{\pi_{k+1}}\left(x_{k}\right)-c_{3} \pi_{k+1} .
$$

Now, consider the scaled penalty function

$$
\frac{1}{\pi} \phi_{\pi}(x)=\frac{1}{\pi} f(x)+v(x),
$$

and note that since $\left\{f_{k}\right\}$ is assumed bounded below and the algorithm is unaffected by adding a constant to $f$, we may assume without loss of generality that $f\left(x_{k}\right) \geq 0$ for all $k$. This assumption and the fact that $\left\{\pi_{k}\right\}$ is nondecreasing, imply that

$$
\frac{1}{\pi_{k+1}} f\left(x_{k}\right)+v\left(x_{k}\right) \leq \frac{1}{\pi_{k}} f\left(x_{k}\right)+v\left(x_{k}\right) .
$$


By the sufficient decrease condition (2.14) we have that, for all $k$,

$$
\frac{1}{\pi_{k+1}} \phi\left(x_{k+1}\right) \leq \frac{1}{\pi_{k+1}} \phi\left(x_{k}\right) .
$$

By combining this expression with (3.30) we have

$$
\frac{1}{\pi_{k+1}} \phi\left(x_{k+1}\right) \leq \frac{1}{\pi_{k}} \phi\left(x_{k}\right)
$$

which shows that the sequence $\left\{\frac{1}{\pi_{k}} f\left(x_{k}\right)+v\left(x_{k}\right)\right\}$ is monotone decreasing for all $k$. Consider now an iterate $x_{k} \in \mathcal{B}^{*}$ at which Step 4(a) is executed. By combining (3.30) with (3.29) we obtain, for such $x_{k}$,

$$
\frac{1}{\pi_{k+1}} f\left(x_{k+1}\right)+v\left(x_{k+1}\right) \leq \frac{1}{\pi_{k}} f\left(x_{k}\right)+v\left(x_{k}\right)-c_{3} .
$$

This contradicts Assumption A1 that implies that the sequence $\left\{\frac{1}{\pi_{k}} f\left(x_{k}\right)+v\left(x_{k}\right)\right\}$ is bounded below. We conclude that Step 4(a) can only be executed finitely often for $x_{k} \in \mathcal{B}^{*}$.

Case (ii) Next, consider Step 4(b). First note that, since $W_{k}$ is positive definite, the lowest value of the quadratic model $q_{k}^{f}(d)$ (defined in $(3.10)$ ) is attained at the Newton step, $-W_{k}^{-1} \nabla f_{k}$. Thus by (3.1) we have

$$
\begin{aligned}
q_{k}^{f}(d) & \geq f_{k}-\nabla f_{k}^{T} W_{k}^{-1} \nabla f_{k}+\frac{1}{2} \nabla f_{k}^{T} W_{k}^{-1} W_{k} W_{k}^{-1} \nabla f_{k} \\
& =f_{k}-\frac{1}{2} \nabla f_{k}^{T} W_{k}^{-1} \nabla f_{k} \\
& \geq f_{k}-\frac{1}{2}\left\|\nabla f_{k}\right\|^{2} / \mu_{\min } .
\end{aligned}
$$

Also, since the linear program (2.8) is constrained by a trust region whose radius cannot exceed $\Delta_{\max }$, we have from $(3.10)$

$$
q_{k}^{f}\left(d_{k}^{\mathrm{LP}}\right) \leq f_{k}+\left\|\nabla f_{k}\right\| \Delta_{\max }+\frac{1}{2} \mu_{\max } \Delta_{\max }^{2} .
$$

By combining (3.31) and (3.32) and recalling that $\left\|\nabla f_{k}\right\|$ is bounded (by Assumption A1), we deduce that there is constant $\nu$ such that, for all $x_{k}$

$$
q_{k}^{f}\left(d^{\mathrm{LP}}\right)-q_{k}^{f}\left(d_{k}\left(\pi_{k}\right)\right) \leq \nu
$$

Now since $d_{k}\left(\pi_{k}\right)$ minimizes $q_{\pi_{k}}$, by (3.10) we have that

$$
q_{k}^{f}\left(d_{k}\left(\pi_{k}\right)\right)+\pi_{k} m_{k}\left(d_{k}\left(\pi_{k}\right)\right) \leq q_{k}^{f}\left(d^{\mathrm{LP}}\right)+\pi_{k} m_{k}\left(d^{\mathrm{LP}}\right) .
$$

Combining this relation with (3.33) gives

$$
\begin{aligned}
\pi_{k}\left[m_{k}(0)-m_{k}\left(d_{k}\left(\pi_{k}\right)\right)\right] & \geq \pi_{k}\left[m_{k}(0)-m_{k}\left(d_{k}^{\mathrm{LP}}\right)\right]-q_{k}^{f}\left(d^{\mathrm{LP}}\right)+q_{k}^{f}\left(d_{k}\left(\pi_{k}\right)\right) \\
& \geq \pi_{k}\left[m_{k}(0)-m_{k}\left(d_{k}^{\mathrm{LP}}\right)\right]-\nu \\
& \geq \pi_{k}\left[m_{k}(0)-m_{k}\left(d_{k}^{\mathrm{LP}}\right)\right]\left(1-\frac{\nu}{\zeta \pi_{k}}\right)
\end{aligned}
$$

because (3.25) implies that $-\nu \geq-\nu\left[m_{k}(0)-m_{k}\left(d_{k}^{\mathrm{LP}}\right)\right] / \zeta$. If the penalty parameter is large enough that

$$
\pi_{k} \geq \frac{\nu}{\zeta\left(1-\epsilon_{1}\right)}
$$


then

$$
m_{k}(0)-m_{k}\left(d_{k}\left(\pi_{k}\right)\right) \geq \epsilon_{1}\left[m_{k}(0)-m_{k}\left(d^{\mathrm{LP}}\right)\right],
$$

and condition (2.12) will be satisfied. Therefore, $\pi_{k}$ cannot be increased infinitely often in Step $4(\mathrm{~b})$, for $x_{k} \in \mathcal{B}^{*}$.

Case (iii) Finally, consider Step 5 of Algorithm I, which enforces condition (2.13). Suppose that $\pi_{+}$is chosen so that

$$
\pi_{+} \geq \frac{\nu}{\zeta\left(1-\epsilon_{1}\right)\left(1-\epsilon_{2} / \epsilon_{1}\right)}
$$

(recall that $\left.\epsilon_{2}<\epsilon_{1}\right)$. If we let $\tilde{\pi}=\pi_{+}\left(1-\epsilon_{2} / \epsilon_{1}\right)$ then the fact that $d(\tilde{\pi})$ is a minimizer of $q^{\tilde{\pi}}$ implies that

$$
q_{k}^{f}(0)-q_{k}^{f}\left(d_{k}(\tilde{\pi})\right)+\pi_{+}\left(1-\epsilon_{2} / \epsilon_{1}\right)\left(m_{k}(0)-m_{k}\left(d_{k}(\tilde{\pi})\right) \geq 0,\right.
$$

and therefore

$$
q_{k}^{f}(0)-q_{k}^{f}(d(\tilde{\pi}))+\pi_{+}\left[m_{k}(0)-m_{k}\left(d_{k}(\tilde{\pi})\right)\right] \geq \frac{\epsilon_{2}}{\epsilon_{1}} \pi_{+}\left[m_{k}(0)-m_{k}\left(d_{k}(\tilde{\pi})\right)\right] .
$$

Thus,

$$
q_{k}^{\pi_{+}}(0)-q_{k}^{\pi_{+}}\left(d_{k}(\tilde{\pi})\right) \geq \epsilon_{2} \pi_{+}\left(m_{k}(0)-m_{k}\left(d_{k}^{\mathrm{LP}}\right)\right)
$$

since $\tilde{\pi}$ satisfies (3.34) and thus (2.12). The fact that $\pi_{+}$satisfies (2.13) follows from noting that $-q_{k}^{\pi_{+}}\left(d_{k}(\tilde{\pi})\right) \leq-q_{k}^{\pi_{+}}\left(d_{k}\left(\pi_{+}\right)\right)$since $d\left(\pi_{+}\right)$is a minimizer of $q^{\pi_{+}}$.

Therefore since any $\pi_{+}$satisfying (3.36) satisfies (2.12) and (2.13) and since $\pi$ increases by at least $\rho$, only a finite number of increases can occur for $x_{k} \in \mathcal{B}^{*}$.

We now consider the behavior of the algorithm in the vicinity of a point that satisfies the well-known Mangasarian-Fromovitz constraint qualification (MFCQ) [25].

Lemma 3.9 Let $x_{*}$ be a point that satisfies MFCQ and suppose that Assumptions I hold. Then, there is a neighborhood $\mathcal{N}$ of $x_{*}$ and a constant $r_{F}$ such that, for any iterate $x_{k} \in \mathcal{N}$, there is a vector $d_{F}\left(x_{k}\right)$ with $\left\|d_{F}\left(x_{k}\right)\right\| \leq r_{F}$ such that $m_{k}\left(d_{F}\left(x_{k}\right)\right)=0$. In addition there are constants $r$ and $\beta$ such that, for $x_{k} \in \mathcal{N}$, the minimizer $d_{k}(\pi)$ of $q_{\pi}^{k}$ satisfies

$$
\left\|d_{k}\right\| \leq r
$$

and such that

$$
q_{k}^{f}\left(d_{1}\right)-q_{k}^{f}\left(d_{2}\right) \leq \beta\left\|d_{1}-d_{2}\right\|
$$

for any vectors $d_{1}, d_{2}$ such that $\left\|d_{1}\right\| \leq r,\left\|d_{2}\right\| \leq r$.

Proof. Let $h(x)$ denote the vector with components $h_{i}(x), i \in \mathcal{E}, g(x)$ the vector with components $g_{i}(x), i \in \mathcal{I}$ and let $\nabla h(x)^{T}$ and $\nabla g(x)^{T}$ denote their Jacobians. Since MFCQ holds at $x_{*}, \nabla h\left(x_{*}\right)$ has full rank and there is a direction $\left\|d_{\mathrm{M}}\right\|<1$ such that

$$
\nabla h\left(x_{*}\right)^{T} d_{\mathrm{M}}=-h\left(x_{*}\right) \text { and } \nabla g\left(x_{*}\right)^{T} d_{\mathrm{M}}+g\left(x_{*}\right)>0 .
$$


Since $\nabla h\left(x_{*}\right)$ has full rank, for any $x$ sufficiently near $x_{*}$ the matrix $\nabla h(x)^{T} \nabla h(x)$ is nonsingular and the vector

$$
d_{\mathrm{F}}(x)=d_{\mathrm{M}}-\nabla h(x)\left(\nabla h(x)^{T} \nabla h(x)\right)^{-1}\left[h(x)+\nabla h(x)^{T} d_{\mathrm{M}}\right]
$$

satisfies

$$
\nabla h(x)^{T} d_{\mathrm{F}}(x)=-h(x)
$$

By continuity of $\nabla h(x)$, the vector $d_{\mathrm{F}}(x)$ is continuous, and the first condition in (3.39) implies that the term in square brackets in (3.40) is small in norm near $x_{*}$. Therefore, from the second condition in (3.39) we have that $\nabla g(x)^{T} d_{\mathrm{F}}(x)+g(x)>0$ for $x$ near $x_{*}$. Thus, for $x_{k}$ in some neighborhood $\mathcal{N}$ of $x_{*}$, we have that $m_{k}\left(d_{\mathrm{F}}\left(x_{k}\right)\right)=0$. Continuity of $d_{\mathrm{F}}(x)$ also implies that there is a constant $r_{\mathrm{F}}$ such that $\left\|d_{\mathrm{F}}\left(x_{k}\right)\right\|<r_{\mathrm{F}}$ for all $x_{k}$ in $\mathcal{N}$.

Since $d_{\mathrm{F}}\left(x_{k}\right)$ is a minimizer of $m_{k}$, we have that $\left\|\bar{d}_{k}\right\| \leq\left\|d_{\mathrm{F}}\left(x_{k}\right)\right\|$, where $\bar{d}_{k}$ is the minimum norm minimizer of $m_{k}$ mentioned in Lemma 3.3. Thus, by (3.7) we have $\left\|d_{k}(\pi)\right\| \leq$ $r$, with $r=\kappa_{1}+\kappa_{2} r_{\mathrm{F}}$.

From (3.10), $\left\|\nabla q_{k}^{f}(d)\right\| \leq\left\|\nabla f\left(x_{k}\right)\right\|+\left\|W_{k}\right\|\|d\|$. The right hand side of this inequality is bounded for all $x_{k} \in \mathcal{N}$ due to the bounds on $d_{1}, d_{2}$ and the boundedness of $W_{k}$ stipulated in Assumptions I . The result (3.38) then follows by a Taylor expansion of $q_{k}^{f}$.

For the following results, we define $\mathcal{A}^{*}$ to be the set of active inequality constraints at $x_{*}$, i.e., $\mathcal{A}^{*}=\left\{i \in \mathcal{I}: g_{i}\left(x_{*}\right)=0\right\}$. The next lemma is a technical result establishing a cone of linearized feasibility with respect to constraints not in $\mathcal{A}^{*}$.

Lemma 3.10 Suppose that Assumptions I hold and that $x_{*}$ is a feasible point with active set $\mathcal{A}^{*}$. Then, there exists a constant $\gamma>0$ and a neighborhood of $x_{*}$, such that for any $x_{k}$ in that neighborhood, for any step $d_{k}$ satisfying

$$
g_{i}\left(x_{k}\right)+\nabla g_{i}\left(x_{k}\right)^{T} d_{k} \geq 0, \text { for all } i \notin \mathcal{A}^{*}
$$

and for any direction $\tilde{d}$, we have

$$
g_{i}\left(x_{k}\right)+\nabla g_{i}\left(x_{k}\right)^{T}\left(\alpha d_{k}+\tau \tilde{d}\right) \geq 0, \text { for all } i \notin \mathcal{A}^{*},
$$

if $\tau>0$ and $\alpha \in(0,1)$ satisfy

$$
\tau\|\tilde{d}\| \leq(1-\alpha) \gamma
$$

Proof. Define $\mathcal{N}^{\prime}$ to be a neighborhood of $x_{*}$ over which $g_{i}(x) \geq \frac{1}{2} g_{i}\left(x_{*}\right)>0$ for all $i \notin \mathcal{A}^{*}$. If $\nabla g_{i}\left(x_{k}\right)=0$ for all $i \notin \mathcal{A}^{*}$ then (3.43) holds trivially. Otherwise, the quantity

$$
\gamma=\min _{i \notin \mathcal{\mathcal { A } ^ { * } , x \in \mathcal { N } ^ { \prime }}} \frac{g_{i}(x)}{\left\|\nabla g_{i}(x)\right\|}
$$

is positive. Multiplying $(3.42)$ by any $\alpha \in(0,1)$ we obtain

$$
g_{i}\left(x_{k}\right)+\nabla g_{i}\left(x_{k}\right)^{T}\left(\alpha d_{k}\right) \geq(1-\alpha) g_{i}\left(x_{k}\right), \text { for all } i \notin \mathcal{A}^{*} .
$$


Now consider the composite direction $\alpha d_{k}+\tau \tilde{d}$, with $\tau \geq 0$ and $\tilde{d}$ an arbitrary direction. We have

$$
g_{i}\left(x_{k}\right)+\nabla g_{i}\left(x_{k}\right)^{T}\left(\alpha d_{k}+\tau \tilde{d}\right) \geq(1-\alpha) g_{i}\left(x_{k}\right)+\tau \nabla g_{i}\left(x_{k}\right)^{T} \tilde{d}, \text { for all } i \notin \mathcal{A}^{*} .
$$

If $x_{k} \in \mathcal{N}^{\prime}$, then $g_{i}\left(x_{k}\right)>0$ for $i \notin \mathcal{A}^{*}$, so that if $\nabla g_{i}\left(x_{k}\right)^{T} \tilde{d} \geq 0$, the right-hand side of (3.46) is nonnegative. If, on the other hand $\nabla g_{i}\left(x_{k}\right)^{T} \tilde{d}<0$ and $\tau$ satisfies (3.44), then

$$
\tau \leq \frac{(1-\alpha) \gamma}{\|\tilde{d}\|} \leq \frac{(1-\alpha) g_{i}\left(x_{k}\right)}{\left\|\nabla g_{i}\left(x_{k}\right)\right\|\|\tilde{d}\|} \leq \frac{(1-\alpha) g_{i}\left(x_{k}\right)}{-\nabla g_{i}\left(x_{k}\right)^{T} \tilde{d}}, \quad i \notin \mathcal{A}^{*}
$$

hence the right-hand side of (3.46) is nonnegative.

The next result shows that, in a vicinity of a feasible point that satisfies the MangasarianFromowitz constraint qualification and for sufficiently large values of the penalty parameter, the step $d_{k}$ generated by the algorithm satisfies the linearized constraints (i.e. the vectors $r, s, t$ in (2.7) are all zero).

Lemma 3.11 Suppose that Algorithm I generates a sequence $\left\{x_{k}\right\}$ that satisfies Assumptions I. Let $x_{*}$ be a cluster point of this sequence such that $v\left(x_{*}\right)=0$, and suppose that MFCQ holds at $x_{*}$. Then for all $x_{k}$ sufficiently close to $x_{*}$ and $\pi_{k}$ sufficiently large, the minimizer $d_{k}$ of $q_{k}^{\pi_{k}}$ satisfies $m_{k}\left(d_{k}\right)=0$.

Proof. As in the proof of Lemma 3.9, we denote by $h(x)$ the vector with components $h_{i}(x), i \in \mathcal{E}$ and denote the Jacobian of $h$ by $\nabla h(x)^{T}$, and similarly for $g(x)$ and $\nabla g(x)^{T}$. Since MFCQ is satisfied at $x_{*}$, we have that $\nabla h\left(x_{*}\right)$ has full rank and there is a direction $\left\|d_{\mathrm{MF}}\right\|<1$ such that

$$
h\left(x_{*}\right)+\nabla h\left(x_{*}\right)^{T} d_{\mathrm{MF}}=0 \quad \text { and } \quad \nabla g\left(x_{*}\right)^{T} d_{\mathrm{MF}}+g\left(x_{*}\right)>0 .
$$

Let us define

$$
d_{\mathrm{M}}(x) \triangleq d_{\mathrm{MF}}-\nabla h(x)\left[\nabla h(x)^{T} \nabla h(x)\right]^{-1} \nabla h(x)^{T} d_{\mathrm{MF}},
$$

which is well defined for $x$ near $x_{*}$ because the matrix $\nabla h(x)^{T} \nabla h(x)$ is nonsingular since it is close to the nonsingular matrix $\nabla h\left(x_{*}\right)^{T} \nabla h\left(x_{*}\right)$. Clearly,

$$
\nabla h(x)^{T} d_{\mathrm{M}}(x)=0 .
$$

Since $x_{*}$ is feasible, we have that $h\left(x_{*}\right)=0$, and thus by the first relation in (3.48) the term $\nabla h(x)^{T} d_{\mathrm{MF}}$ is close to zero for $x_{k}$ near $x_{*}$ - and this implies that $d_{\mathrm{M}}(x)$ is close to $d_{\mathrm{MF}}$. Since $\nabla h$ is a continuous function, $d_{\mathrm{M}}(x)$ varies continuously with $x$, and therefore by the second relation in (3.48) there is a constant $\sigma>0$ such that for all $x_{k}$ near $x_{*}$, the vector $d_{\mathrm{M}}^{k} \triangleq d_{\mathrm{M}}\left(x_{k}\right)$ satisfies

$$
\nabla g_{i}\left(x_{k}\right)^{T} d_{\mathrm{M}}^{k}>\sigma \quad \text { for all } i \in \mathcal{A}^{*},
$$

where, as before, $\mathcal{A}^{*}$ denotes the set of active inequality constraints at $x_{*}$. 
Let us define $\underline{g}=\frac{1}{2} \min _{j \notin \mathcal{A}^{*}}\left\{g_{j}\left(x_{*}\right)\right\}$. Then, for $x_{k}$ sufficiently near $x_{*}$, we have that $v\left(x_{k}\right)<\epsilon$ where $\epsilon>0$ is a constant that may additionally be chosen sufficiently small to satisfy both

$$
g_{j}\left(x_{k}\right) \geq \underline{g}-\epsilon>0 \text { for } j \notin \mathcal{A}^{*}, \text { and } 2 \epsilon<\underline{g} .
$$

We denote by $\mathcal{N}$ a neighborhood of $x_{*}$ contained in the neighborhoods given by Lemmas 3.9 and 3.10, and such that for all $x_{k} \in \mathcal{N}$, conditions (3.50), (3.51) and (3.52) hold and $v\left(x_{k}\right)<\epsilon$. Let us re-write $(2.4)$ as

$$
m_{k}(d)=\sum_{i \notin \mathcal{A}^{*}}\left[g_{i}\left(x_{k}\right)+\nabla g_{i}\left(x_{k}\right)^{T} d\right]^{-}+\sum_{i \in \mathcal{A}^{*}}\left[g_{i}\left(x_{k}\right)+\nabla g_{i}\left(x_{k}\right)^{T} d\right]^{-}+\sum_{i \in \mathcal{E}}\left|h_{i}\left(x_{k}\right)+\nabla h_{i}\left(x_{k}\right)^{T} d\right| .
$$

The proof proceeds in three stages; each shows that one of the summations is zero for any $x_{k} \in \mathcal{N}$ and for sufficiently large $\pi_{k}$.

Part (i) Let $d_{k}$ minimize $q_{k}^{\pi_{k}}$, and suppose by way of contradiction that the first summation is nonzero for $x_{k} \in \mathcal{N}$. Then,

$$
g_{j}\left(x_{k}\right)+\nabla g_{j}\left(x_{k}\right)^{T} d_{k}<0 \quad \text { for some } j \notin \mathcal{A}^{*} .
$$

By (3.52), we have that $g_{j}\left(x_{k}\right)>0$ for $x_{k} \in \mathcal{N}$, and since (3.54) also holds, we know that there exists $\alpha \in(0,1)$ such that

$$
g_{j}\left(x_{k}\right)+\alpha \nabla g_{j}\left(x_{k}\right)^{T} d_{k}=0 .
$$

It follows that

$$
\alpha\left(g_{j}\left(x_{k}\right)+\nabla g_{j}\left(x_{k}\right)^{T} d\right)=-(1-\alpha) g_{j}\left(x_{k}\right),
$$

which together with (3.52) and the condition $\alpha<1$ implies

$$
g_{j}\left(x_{k}\right)+\nabla g_{j}\left(x_{k}\right)^{T} d_{k} \leq-(1-\alpha)(\underline{g}-\epsilon) .
$$

Define the function

$$
\left.a_{j}^{k}(d) \triangleq m_{k}(d)-\left[g_{j}\left(x_{k}\right)+\nabla g_{j}\left(x_{k}\right)^{T} d\right)\right]^{-},
$$

which consists of excluding the $j$ th inequality term from (3.53) (and therefore $a_{j}^{k}\left(d_{k}\right) \geq 0$ ). For $j$ satisfying (3.54), we have

$$
a_{j}^{k}\left(d_{k}\right)=m_{k}\left(d_{k}\right)+\left(g_{j}\left(x_{k}\right)+\nabla g_{j}\left(x_{k}\right)^{T} d_{k}\right) .
$$

Clearly, $a_{j}^{k}$ is a convex function, which implies that for any $d_{k}$

$$
a_{j}^{k}\left(\alpha d_{k}\right) \leq(1-\alpha) a_{j}^{k}(0)+\alpha a_{j}^{k}\left(d_{k}\right)
$$

and thus

$$
a_{j}^{k}\left(\alpha d_{k}\right)-a_{j}^{k}\left(d_{k}\right) \leq(1-\alpha)\left(a_{j}^{k}(0)-a_{j}^{k}\left(d_{k}\right)\right) \leq(1-\alpha) \epsilon,
$$


since by $(3.57) a_{j}^{k}(0)=m_{k}(0)=v\left(x_{k}\right)<\epsilon$, and $a_{j}^{k}\left(d_{k}\right) \geq 0$. Now, we have from (3.57), (3.55), (3.58), (3.59) and (3.56) that

$$
\begin{aligned}
m\left(\alpha d_{k}\right)-m\left(d_{k}\right) & =a_{j}^{k}\left(\alpha d_{k}\right)-a_{j}^{k}\left(d_{k}\right)+\left(g_{j}\left(x_{k}\right)+\nabla g_{j}\left(x_{k}\right)^{T} d_{k}\right) \\
& \leq(1-\alpha) \epsilon-(1-\alpha)(\underline{g}-\epsilon) \\
& =(1-\alpha)(2 \epsilon-\underline{g}) .
\end{aligned}
$$

Finally, by Lemma 3.9 and since $q_{k}^{\pi_{k}}\left(d_{k}\right)=q_{k}^{f}\left(d_{k}\right)+\pi_{k} m_{k}\left(d_{k}\right)$,

$$
q_{k}^{\pi_{k}}\left(\alpha d_{k}\right)-q_{k}^{\pi_{k}}\left(d_{k}\right) \leq(1-\alpha) \beta r+\pi_{k}(1-\alpha)(2 \epsilon-\underline{g}) .
$$

By (3.52), $2 \epsilon-\underline{g}<0$, and if $\pi_{k}>\beta r /(\underline{g}-2 \epsilon)$, we have that $q_{k}^{\pi_{k}}\left(\alpha d_{k}\right)<q_{k}^{\pi_{k}}\left(d_{k}\right)$, which contradicts the fact that $d_{k}$ is the minimizer of $q_{k}^{\pi_{k}}$. Therefore, for $x_{k} \in \mathcal{N}$ and for $\pi_{k}$ sufficiently large, there cannot exist an index $j$ satisfying (3.54), and thus the first summation in $(3.53)$ is zero.

Part (ii) Next, suppose that the step $d_{k}$ that minimizes $q_{k}^{\pi_{k}}$ is such that the second sum in (3.53) is nonzero for $x_{k} \in \mathcal{N}$, while the first sum is zero. Then

$$
g_{\ell}\left(x_{k}\right)+\nabla g_{\ell}\left(x_{k}\right)^{T} d_{k}<0 \text { for some } \ell \in \mathcal{A}^{*} .
$$

As above, consider the linearized model of the constraints other than $\ell$ :

$$
\left.a_{\ell}^{k}(d)=m_{k}(d)-\left[g_{\ell}\left(x_{k}\right)+\nabla g_{\ell}\left(x_{k}\right)^{T} d\right)\right]^{-} .
$$

By (3.50), (3.51) we have that the vector $d_{\mathrm{M}}^{k}=d_{\mathrm{M}}\left(x_{k}\right)$ satisfies $\nabla h_{i}\left(x_{k}\right)^{T} d_{\mathrm{M}}^{k}=0, i \in \mathcal{E}$ and $\nabla g_{i}\left(x_{k}\right)^{T} d_{\mathrm{M}}^{k} \geq \sigma, i \in \mathcal{A}^{*}$; furthermore, for

$$
\tau<(1-\alpha) \gamma
$$

we have by Lemma 3.10 that (3.43) is satisfied for $\tilde{d}=d_{\mathrm{M}}^{k}$ and for $i \notin \mathcal{A}^{*}$, (recall that $\left.\left\|d_{\mathrm{M}}\right\|<1\right)$. These observations show that each of the terms in (3.53) is not larger for $d=\alpha d_{k}+\tau d_{\mathrm{M}}^{k}$ than for $d=\alpha d_{k}$, and the same is true for $a_{\ell}^{k}$ since $a_{\ell}^{k}$ consists of all but one of the terms in $m_{k}$. Thus,

$$
a_{\ell}^{k}\left(\alpha d_{k}+\tau d_{\mathrm{M}}^{k}\right) \leq a_{\ell}^{k}\left(\alpha d_{k}\right) \leq(1-\alpha) a_{\ell}^{k}(0)+a_{\ell}^{k}\left(d_{k}\right),
$$

where the second inequality follows from the convexity of $a_{\ell}^{k}$ and the condition $\alpha \in(0,1)$. Since $\ell \in \mathcal{A}^{*}$, we also have from (3.51) that

$$
g_{\ell}\left(x_{k}\right)+\nabla g_{\ell}\left(x_{k}\right)^{T}\left(\alpha d_{k}+\tau d_{\mathrm{M}}^{k}\right) \geq g_{\ell}\left(x_{k}\right)+\alpha \nabla g_{\ell}\left(x_{k}\right)^{T} d_{k}+\tau \sigma .
$$

If we choose $\tau>0$ small enough so that

$$
\tau \sigma \leq-\left(g_{\ell}\left(x_{k}\right)+\nabla g_{\ell}\left(x_{k}\right)^{T} d_{k}\right)
$$

then by $(3.61)$

$$
\begin{aligned}
{\left[g_{\ell}\left(x_{k}\right)+\nabla g_{\ell}\left(x_{k}\right)^{T} d_{k}+\tau \sigma\right]^{-} } & =-\left(g_{\ell}\left(x_{k}\right)+\nabla g_{\ell}\left(x_{k}\right)^{T} d_{k}\right)-\tau \sigma \\
& =\left[g_{\ell}\left(x_{k}\right)+\nabla g_{\ell}\left(x_{k}\right)^{T} d_{k}\right]^{-}-\tau \sigma .
\end{aligned}
$$


By making use of $(3.65)$, the fact that $[\cdot]^{-}$is a non-increasing convex function, the condition $\alpha<1$ and (3.67) we have

$$
\begin{aligned}
{\left[g_{\ell}\left(x_{k}\right)+\nabla g_{\ell}\left(x_{k}\right)^{T}\left(\alpha d_{k}+\tau d_{\mathrm{M}}^{k}\right)\right]^{-} } & \leq\left[g_{\ell}\left(x_{k}\right)+\alpha \nabla g_{\ell}\left(x_{k}\right)^{T} d_{k}+\tau \sigma\right]^{-} \\
& \leq(1-\alpha)\left[g_{\ell}\left(x_{k}\right)+\tau \sigma\right]^{-}+\alpha\left[g_{\ell}\left(x_{k}\right)+\nabla g_{\ell}\left(x_{k}\right)^{T} d_{k}+\tau \sigma\right]^{-} \\
& \leq(1-\alpha)\left[g_{\ell}\left(x_{k}\right)\right]^{-}+\alpha\left[g_{\ell}\left(x_{k}\right)+\nabla g_{\ell}\left(x_{k}\right)^{T} d_{k}+\tau \sigma\right]^{-} \\
& \leq(1-\alpha)\left[g_{\ell}\left(x_{k}\right)\right]^{-}+\left[g_{\ell}\left(x_{k}\right)+\nabla g_{\ell}\left(x_{k}\right)^{T} d_{k}+\tau \sigma\right]^{-} \\
& \leq(1-\alpha)\left[g_{\ell}\left(x_{k}\right)\right]^{-}+\left[g_{\ell}\left(x_{k}\right)+\nabla g_{\ell}\left(x_{k}\right)^{T} d_{k}\right]^{-}-\tau \sigma .(3.68)
\end{aligned}
$$

Now, using (3.62) to decompose $m_{k}$ and then applying (3.64) and (3.68), we obtain

$$
\begin{aligned}
m_{k}\left(\alpha d_{k}+\tau d_{\mathrm{M}}^{k}\right) & =a_{\ell}^{k}\left(\alpha d_{k}+\tau d_{\mathrm{M}}^{k}\right)+\left[g_{\ell}\left(x_{k}\right)+\nabla g_{\ell}\left(x_{k}\right)^{T}\left(\alpha d_{k}+\tau d_{\mathrm{M}}^{k}\right)\right]^{-} \\
& \leq(1-\alpha) a_{\ell}^{k}(0)+a_{\ell}^{k}\left(d_{k}\right)+(1-\alpha)\left[g_{\ell}\left(x_{k}\right)\right]^{-}+\left[g_{\ell}\left(x_{k}\right)+\nabla g_{\ell}\left(x_{k}\right)^{T} d_{k}\right]^{-}-\tau \sigma \\
& \leq(1-\alpha) m_{k}(0)+m_{k}\left(d_{k}\right)-\tau \sigma \\
& \leq(1-\alpha) \epsilon+m_{k}\left(d_{k}\right)-\tau \sigma
\end{aligned}
$$

for any $\alpha \in[0,1]$, and $\tau>0$ satisfying (3.63) and (3.66). Since, by Lemma 3.9, $\left\|d_{k}\right\| \leq r$, we may also choose $\tau$ small enough that $\left\|\alpha d_{k}+\tau d_{\mathrm{M}}^{k}\right\| \leq 2 r$. Then, if we additionally require that (3.63) holds with equality, we have from (3.11), Lemma 3.9 and (3.69) that

$$
\begin{aligned}
q_{\pi}^{k}\left(\alpha d_{k}+\tau d_{\mathrm{M}}^{k}\right)-q_{\pi}^{k}\left(d_{k}\right) & \leq q_{f}^{k}\left(\alpha d_{k}+\tau d_{\mathrm{M}}^{k}\right)-q_{k}^{f}\left(d_{k}\right)-\pi(\tau \sigma-(1-\alpha) \epsilon) \\
& \leq \beta\left\|(\alpha-1) d_{k}+\tau d_{\mathrm{M}}^{k}\right\|-\pi(\tau \sigma-(1-\alpha) \epsilon) \\
& \leq\left(\frac{r}{\gamma}+\left\|d_{\mathrm{M}}^{k}\right\|\right) \beta \tau-\pi \tau\left(\sigma-\frac{\epsilon}{\gamma}\right)
\end{aligned}
$$

where we have applied Lemma 3.10 with the condition that $\tau$ is small enough that $\alpha \in(0,1)$. If we choose $\epsilon<\gamma \sigma / 2$, then for $\pi>2 \beta\left(r / \gamma+\left\|d_{\mathrm{M}}^{k}\right\|\right) / \sigma$ the right hand side is negative, which is not possible because $d_{k}$ is the minimizer of $q_{\pi}^{k}$. Therefore the inequality (3.54) cannot hold for $x_{k} \in \mathcal{N}$ and $\pi_{k}$ large enough.

Part (iii) Last, suppose that the step $d_{k}$ that minimizes $q_{k}^{\pi_{k}}$ satisfies all the linearized inequalities (so that the first two summations in (3.53) are zero), but is such that $h\left(x_{k}\right)+$ $\nabla h\left(x_{k}\right)^{T} d_{k} \neq 0$. Then

$$
m\left(d_{k}\right)=\left\|h\left(x_{k}\right)+\nabla h\left(x_{k}\right)^{T} d_{k}\right\| .
$$

Consider taking a step from $d_{k}$ in the direction

$$
p=-\nabla h\left(x_{k}\right)\left[\nabla h\left(x_{k}\right)^{T} \nabla h\left(x_{k}\right)\right]^{-1}\left(h\left(x_{k}\right)+\nabla h\left(x_{k}\right)^{T} d_{k}\right)+\theta d_{\mathrm{M}}^{k},
$$

for some $\theta>0$. We have from (3.50) that, for any $\alpha, \tau$, such that $\tau<\alpha<1$,

$$
\begin{aligned}
h\left(x_{k}\right)+\nabla h\left(x_{k}\right)^{T}\left(\alpha d_{k}+\tau p\right) & =h\left(x_{k}\right)+\nabla h\left(x_{k}\right)^{T} \alpha d_{k}-\tau\left[h\left(x_{k}\right)+\nabla h\left(x_{k}\right)^{T} d_{k}\right], \\
& =(\alpha-\tau)\left[h\left(x_{k}\right)+\nabla h\left(x_{k}\right)^{T} d_{k}\right]+(1-\alpha) h\left(x_{k}\right) .
\end{aligned}
$$


Since for $x_{k} \in \mathcal{N}$ we have $\left\|h\left(x_{k}\right)\right\| \leq \epsilon$, and since $\alpha_{k} \leq 1$, we obtain

$$
\left\|h\left(x_{k}\right)+\nabla h\left(x_{k}\right)^{T}\left(\alpha d_{k}+\tau p\right)\right\| \leq(1-\tau)\left\|h\left(x_{k}\right)+\nabla h\left(x_{k}\right)^{T} d_{k}\right\|+(1-\alpha) \epsilon .
$$

By Assumption A1, the fact that $\nabla h(x)$ has full rank near $x_{*}$ and (3.51), we have that for $i \in \mathcal{A}^{*}$, there is a constant $C_{1}$ such that

$$
\begin{aligned}
\nabla g_{i}\left(x_{k}\right)^{T} p & \geq-\nabla g_{i}\left(x_{k}\right)^{T} \nabla h\left(x_{k}\right)\left[\nabla h\left(x_{k}\right)^{T} \nabla h\left(x_{k}\right)\right]^{-1}\left(h\left(x_{k}\right)+\nabla h\left(x_{k}\right)^{T} d_{k}\right)+\theta \sigma \\
& \geq-C_{1}\left\|h\left(x_{k}\right)+\nabla h\left(x_{k}\right)^{T} d_{k}\right\|+\theta \sigma \\
& =\frac{2}{3} \theta \sigma>0,
\end{aligned}
$$

provided we choose $\theta=3 C_{1}\left\|h\left(x_{k}\right)+\nabla h\left(x_{k}\right)^{T} d_{k}\right\| / \sigma$. This bound and the fact that [ $]^{-}$ is a non-increasing convex function, imply that for all $i \in \mathcal{A}^{*}$

$$
\begin{aligned}
{\left[g_{i}\left(x_{k}\right)+\nabla g_{i}\left(x_{k}\right)^{T}\left(\alpha d_{k}+\tau p\right)\right]^{-} } & \leq\left[g_{i}\left(x_{k}\right)+\nabla g_{i}\left(x_{k}\right)^{T} \alpha d_{k}\right]^{-} \\
& \leq(1-\alpha)\left[g_{i}\left(x_{k}\right)\right]^{-}+\alpha\left[g_{i}\left(x_{k}\right)+\nabla g_{i}\left(x_{k}\right)^{T} d_{k}\right]^{-} \\
& \leq(1-\alpha)\left[g_{i}\left(x_{k}\right)\right]^{-}
\end{aligned}
$$

where the last inequality follows from the assumption that $d_{k}$ satisfies all linearized inequalities. Our choice of $\theta$ implies that the length of vector $p$ is bounded as follows,

$$
\begin{aligned}
\|p\| & \leq C_{2}\left\|h\left(x_{k}\right)+\nabla h\left(x_{k}\right)^{T} d_{k}\right\|+\theta\left\|d_{\mathrm{M}}^{k}\right\| \\
& =\left\|h\left(x_{k}\right)+\nabla h\left(x_{k}\right)^{T} d_{k}\right\|\left(C_{2}+3 C_{1}\left\|d_{\mathrm{M}}^{k}\right\| / \sigma\right) \\
& \leq C_{3}\left\|h\left(x_{k}\right)+\nabla h\left(x_{k}\right)^{T} d_{k}\right\|,
\end{aligned}
$$

for suitable constants $C_{2}$ and $C_{3}$. If we choose $\tau$ and $\alpha \in(0,1)$ to satisfy

$$
\tau C_{3}\left\|h\left(x_{k}\right)+\nabla h\left(x_{k}\right)^{T} d_{k}\right\|=(1-\alpha) \gamma,
$$

then by Lemma 3.10 we have that condition (3.43) is satisfied for $\tilde{d}=p$. This observation together with (3.73), (3.74) and the convexity of $[\cdot]^{-}$, yield

$$
\begin{aligned}
m_{k}\left(\alpha d_{k}+\tau p\right)= & \left\|h\left(x_{k}\right)+\nabla h\left(x_{k}\right)^{T}\left(\alpha d_{k}+\tau p\right)\right\|+\sum_{i \notin \mathcal{A}^{*}}\left[g_{i}\left(x_{k}\right)+\nabla g_{i}\left(x_{k}\right)^{T}\left(\alpha d_{k}+\tau p\right)\right]^{-} \\
& +\sum_{i \in \mathcal{A}^{*}}\left[g_{i}\left(x_{k}\right)+\nabla g_{i}\left(x_{k}\right)^{T}\left(\alpha d_{k}+\tau p\right)\right]^{-} \\
\leq & \left\|h\left(x_{k}\right)+\nabla h\left(x_{k}\right)^{T}\left(\alpha d_{k}+\tau p\right)\right\|+\sum_{i \in \mathcal{A}^{*}}\left[g_{i}\left(x_{k}\right)+\nabla g_{i}\left(x_{k}\right)^{T}\left(\alpha d_{k}+\tau p\right)\right]^{-} \\
\leq & (1-\tau)\left\|h\left(x_{k}\right)+\nabla h\left(x_{k}\right)^{T} d_{k}\right\|+(1-\alpha) \epsilon+(1-\alpha) \sum_{i \in \mathcal{A}^{*}}\left[g_{i}\left(x_{k}\right)\right]^{-} \\
\leq & m\left(d_{k}\right)-\tau\left\|h\left(x_{k}\right)+\nabla h\left(x_{k}\right)^{T} d_{k}\right\|+2(1-\alpha) \epsilon,
\end{aligned}
$$

where the last inequality follows from (3.70) and the condition $m_{k}(0)<\epsilon$. It follows from this inequality, the Lipschitz condition (3.38) of Lemma 3.9, (3.37) and (3.75), that

$$
\begin{aligned}
q_{\pi}^{k}\left(\alpha d_{k}+\tau p\right)-q_{\pi}^{k}\left(d_{k}\right) & \leq q_{k}^{f}\left(\alpha d_{k}+\tau p\right)-q_{k}^{f}\left(d_{k}\right)+\pi\left[-\tau\left\|h\left(x_{k}\right)+\nabla h\left(x_{k}\right)^{T} d_{k}\right\|+2(1-\alpha) \epsilon\right] \\
& \leq \beta\left(\|\tau p\|+(1-\alpha)\left\|d_{k}\right\|\right)+\pi\left[-\tau\left\|h\left(x_{k}\right)+\nabla h\left(x_{k}\right)^{T} d_{k}\right\|+2(1-\alpha) \epsilon\right] \\
& \left.\leq \beta(1-\alpha) r+\left(\beta C_{3}-\pi\right) \tau\left\|h\left(x_{k}\right)+\nabla h\left(x_{k}\right)^{T} d_{k}\right\|+2 \pi(1-\alpha) \epsilon\right] \\
& \leq\left(\beta C_{3}-\pi\right) \tau\left\|h\left(x_{k}\right)+\nabla h\left(x_{k}\right)^{T} d_{k}\right\|+(\beta r+2 \pi \epsilon)(1-\alpha) .
\end{aligned}
$$


Since we have chosen $\tau$ and $\alpha$ to satisfy (3.76), we have

$$
\begin{aligned}
q_{\pi}^{k}\left(\alpha d_{k}+\tau p\right)-q_{\pi}^{k}\left(d_{k}\right) & \leq\left[\left(\beta C_{3}-\pi\right)+(\beta r+2 \pi \epsilon) C_{3} / \gamma\right] \tau\left\|h\left(x_{k}\right)+\nabla h\left(x_{k}\right)^{T} d_{k}\right\| \\
& \leq\left[\left(\beta C_{3}+\beta r C_{3} / \gamma\right)+\pi\left(-1+2 C_{3} \epsilon / \gamma\right)\right] \tau\left\|h\left(x_{k}\right)+\nabla h\left(x_{k}\right)^{T} d_{k}\right\| .
\end{aligned}
$$

If the neighborhood of $x_{*}$ is chosen small enough that $\epsilon C_{3} / \gamma<1 / 4$, then for $\pi>2 \beta C_{3}(1+$ $r / \gamma)$, we have that $q_{\pi}^{k}\left(\alpha d_{k}+\tau p\right)-q_{\pi}^{k}\left(d_{k}\right)<0$, which contradicts the fact that $d_{k}$ is the minimizer of $q_{\pi_{k}}$. Therefore, we must have that $h\left(x_{k}\right)+\nabla h\left(x_{k}\right)^{T} d_{k}=0$, and this concludes the proof.

We can now prove the main convergence result of this paper.

Theorem 3.12 Suppose that Algorithm I generates an infinite sequence of iterates $\left\{x_{k}\right\}$ and that Assumptions I hold. Then,

(a) If $\left\{\pi_{k}\right\}$ is bounded, any limit point of $\left\{x_{k}\right\}$ is either a KKT point of the nonlinear program (2.1) or is an infeasible stationary point;

(b) If $\left\{\pi_{k}\right\} \rightarrow \infty$, then either there is a limit point $x_{*}$ that is an infeasible stationary point or there is a feasible limit point $x_{*}$ where MFCQ fails.

Proof. Part (a) follows directly from Theorem 3.7.

To prove part (b), when $\left\{\pi_{k}\right\} \rightarrow \infty$, consider an infinite subsequence $x_{k}, k \in \mathcal{K}$ over which $\pi_{k}$ is increased without bound. Since by Assumption A1 this sequence is bounded, it has at least one limit point, say $x_{*}$.

Suppose that $v\left(x_{*}\right)>0$. Then by Lemma 3.8, if $m_{*}(0)-m_{*}\left(d^{\mathrm{LP}}\right)>0$, the penalty parameter $\pi$ can be increased only finitely often in a neighborhood of $x_{*}$. So the fact that $x_{*}$ is a limit point of the sequence $x_{k}, k \in \mathcal{K}$ defined above, implies that $m_{*}(0)-m_{*}\left(d^{\mathrm{LP}}\right)=0$, i.e. that $x_{*}$ is an infeasible stationary point (see Theorem 3.2 (c)).

Suppose on the other hand that $v\left(x_{*}\right)=0$. If $x_{*}$ satisfies MFCQ, then by Lemma 3.11 we have that, for $\pi_{k}$ sufficiently large, $m_{k}\left(d_{k}\right)=0$, for all $x_{k}$ in a neighborhood of $x_{*}$. By Step 2 of Algorithm I, this implies that once $\pi_{k}$ is large enough it will no longer be increased in this neighborhood of $x_{*}$. This contradicts our assumption that $x_{*}$ is the limit point of a subsequence over which the penalty parameter is increased without bound. Therefore, MFCQ must fail at $x_{*}$.

\section{$4 \quad$ Numerical Experiments}

We developed a MATLAB implementation of Algorithm I and tested its performance on several difficult situations. We present results on five small-dimensional examples that exhibit inconsistent constraint linearizations at some iterate or that fail to satisfy the linear independence constraint qualification at the solution. One of the test problems is infeasible. The analysis in this paper indicates that the algorithm should be very robust, and these examples are chosen to test that robustness in cases where the theory applies and in cases that go beyond the theory. Important issues related to the efficient sparse implementation of Algorithm I are not addressed here as they lie outside the scope of this paper. 
To solve the subproblems in Algorithm I, we employed the codes provided by the MATLAB Optimization toolbox. The linear program (2.9) was solved using linprog and the quadratic program (2.7), using quadprog.

We mentioned in Section 2 that the trust region radius $\Delta_{k}$ used in the linear program (2.9) is not crucial; in fact the convergence properties established in the previous section hold even if this radius is kept constant. In practice, however, it may be advantageous to choose $\Delta_{k}$ based on local information of the problem, and in our implementation this choice is based on the most recently accepted step. Given the search direction $d_{k}$ and the step length $\alpha_{k}$ at the end of iteration $k$ of Algorithm I, we compute

$$
a_{r e d}^{k}=\phi_{\pi_{+}}\left(x_{k}\right)-\phi_{\pi_{+}}\left(x_{k}+\alpha_{k} d_{k}\right), \quad p_{r e d}^{k}=q_{k}^{\pi_{+}}(0)-q_{k}^{\pi_{+}}\left(\alpha_{k} d_{k}\right)
$$

and update the LP trust region radius as follows

\section{Procedure for Updating $\Delta_{k}$.}

Initial data: $\eta_{1}<\eta_{2} \in(0,1), \Delta_{\min }, \Delta_{\max }>0$.

If $a_{r e d}^{k}<\eta_{1} p_{r e d}^{k}$ set $\Delta_{k+1}=\frac{1}{2}\left\|\alpha_{k} d_{k}\right\|$

else if $a_{\text {red }}^{k}>\eta_{2} p_{\text {red }}^{k}$ set $\Delta_{k+1}=2\left\|\alpha_{k} d_{k}\right\|$

else

$$
\text { set } \Delta_{k+1}=\left\|\alpha_{k} d_{k}\right\|
$$

Set $\Delta_{k+1}=\operatorname{mid}\left(\Delta_{\min }, \Delta_{k+1}, \Delta_{\max }\right)$.

The initial penalty $\pi_{1}$ is set to 1 in all tests. Algorithm I stops in Step 1 and reports optimality if the infinity norm of the KKT error is less than $10^{-6}$. Convergence to an infeasible stationary point is reported if Algorithm I executes Step 3 and $m_{k}(0)-m_{k}\left(d^{\mathrm{LP}}\right)<$ $10^{-15}$. We multiply $\pi$ by 10 whenever it is increased and set $\Delta_{\min }, \Delta_{1}, \Delta_{\max }$ to $10^{-3}, 1,10^{3}$, respectively. The rest of the parameters are chosen as $\tau=0.5, \eta=10^{-4}, \epsilon_{1}=\epsilon_{2}=0.1$ and $\eta_{1}=0.25, \eta_{2}=0.75$.

The first example illustrates the behavior of Algorithm I when the linearizations of the constraints are inconsistent. In this situation, some SQP methods trigger a switch and revert either to a feasibility restoration phase in which the objective function is ignored, as in FILTERSQP [15], or to an elastic mode ( $\ell_{1}$ minimization) phase, as in SNOPT [18]. There is no switch in Algorithm I, which always takes steps based on the penalty function $\phi_{\pi}$. An important difference between Algorithm I and the penalty update strategy in SNOPT is that the latter follows a traditional approach in which the penalty parameter is held fixed during the course of the minimization, and is only increased when a stationary point of the penalty function is approximated. Algorithm I, on the other hand, employs the steering rules described in $\S 2$ for updating $\pi$.

Example 1. The problem

$$
\begin{aligned}
\operatorname{minimize} & x_{1} \\
\text { subject to } x_{1}^{2}+1-x_{2} & =0, \\
x_{1}-1-x_{3} & =0, \\
x_{2} \geq 0, x_{3} & \geq 0
\end{aligned}
$$


was introduced by Wächter and Biegler [29] to show that a class of line search interior-point methods may converge to a non-stationary point. We use the starting point $(-3,1,1)$; the solution is $x_{*}=(1,2,0)$. The output is summarized in Table 1 , which reports the iteration number (it) the value of the penalty parameter $\pi_{k}$, the trust region $\Delta_{k-1}$ used to generate the latest iterate, the number of quadratic programs (QPs) solved at the current iterate, and the number of linear programs (LPs) solved (0 or 1$)$. The table also prints the values of the first two components of $x$, the KKT and feasibility errors, as well as the value of the objective function $f$ and the penalty function $\phi$.

Table 1: Output for Example 1.

\begin{tabular}{|c|c|c|c|c|c|c|c|c|c|c|}
\hline it & $\pi_{k}$ & $\Delta_{k-1}$ & QPs & LPs & $x_{1}$ & $x_{2}$ & $\operatorname{KKT}(x)$ & feas $(x)$ & $f(x)$ & $\phi_{\pi_{+}}(x)$ \\
\hline \hline 0 & 1 & & & & -3 & 1 & $9.00 \mathrm{E}+00$ & $1.40 \mathrm{E}+01$ & $-3.00 \mathrm{E}+00$ & $1.10 \mathrm{E}+01$ \\
\hline 1 & 1 & $1.00 \mathrm{E}+00$ & 1 & 1 & -1.5405 & 1.2432 & $2.54 \mathrm{E}+00$ & $4.67 \mathrm{E}+00$ & $-1.54 \mathrm{E}+00$ & $3.13 \mathrm{E}+00$ \\
\hline 2 & 1 & $2.92 \mathrm{E}+00$ & 1 & 1 & -0.9428 & 1.5316 & $1.94 \mathrm{E}+00$ & $2.30 \mathrm{E}+00$ & $-9.43 \mathrm{E}-01$ & $1.36 \mathrm{E}+00$ \\
\hline 3 & 1 & $1.20 \mathrm{E}+00$ & 1 & 1 & -0.8115 & 1.6414 & $1.81 \mathrm{E}+00$ & $1.83 \mathrm{E}+00$ & $-8.12 \mathrm{E}-01$ & $1.02 \mathrm{E}+00$ \\
\hline 4 & 1 & $2.63 \mathrm{E}-01$ & 1 & 1 & -0.8043 & 1.6468 & $1.80 \mathrm{E}+00$ & $1.80 \mathrm{E}+00$ & $-8.04 \mathrm{E}-01$ & $1.00 \mathrm{E}+00$ \\
\hline 5 & 1 & $1.45 \mathrm{E}-02$ & 2 & 1 & -0.2924 & 0.8234 & $6.10 \mathrm{E}+00$ & $1.55 \mathrm{E}+00$ & $-2.92 \mathrm{E}-01$ & $1.53 \mathrm{E}+01$ \\
\hline 6 & 10 & $8.23 \mathrm{E}-01$ & 1 & 0 & 0.3538 & 0.5766 & $8.25 \mathrm{E}+00$ & $1.19 \mathrm{E}+00$ & $3.54 \mathrm{E}-01$ & $1.23 \mathrm{E}+01$ \\
\hline 7 & 10 & $6.46 \mathrm{E}-01$ & 1 & 1 & 1 & 1.4265 & $1.23 \mathrm{E}+01$ & $5.73 \mathrm{E}-01$ & $1.00 \mathrm{E}+00$ & $6.73 \mathrm{E}+00$ \\
\hline 8 & 10 & $1.70 \mathrm{E}+00$ & 1 & 0 & 1 & 2 & $5.73 \mathrm{E}-01$ & $2.69 \mathrm{E}-16$ & $1.00 \mathrm{E}+00$ & $1.00 \mathrm{E}+00$ \\
\hline 9 & 10 & $1.15 \mathrm{E}+00$ & 1 & 0 & 1 & 2 & $2.22 \mathrm{E}-15$ & $2.69 \mathrm{E}-16$ & $1.00 \mathrm{E}+00$ & $1.00 \mathrm{E}+00$ \\
\hline
\end{tabular}

The linearized constraints are not satisfied in the first few iterations, i.e., if $m_{k}\left(d_{k}\right)>0$ and Algorithm I therefore solves the linear feasibility LP subproblem. Note that progress toward the solution is made during these initial iterations. The penalty parameter is increased only once, at iteration 5 , meaning that the initial penalty $\pi=1$ adequately relaxed the constraints at the earlier iterations. At iteration 5, the search direction has to be recomputed and hence two QPs are solved. Accurate optimal values for the primal variables are found at iteration 8, but Algorithm I performs one extra iteration to determine the correct final multipliers.

Example 2. The problem

$$
\begin{aligned}
\operatorname{minimize}\left(x_{2}-1\right)^{2} & \\
\text { subject to } x_{1}^{2} & =0 \\
x_{1}^{3} & =0
\end{aligned}
$$

is presented in Fletcher et al. [17] and is also discussed by Chen and Goldfarb [10]. MFCQ is violated at the solution $x_{*}=(0,1)$, and the linearized constraints are inconsistent at every infeasible point.

Fletcher et al. [17] mention that, starting from the infeasible point $(1,0)$, a feasibility restoration phase is likely to converge to $(0,0)$, which is not the solution of the problem. We ran the FILTERSQP solver [14] and observed that it did indeed converge to $(0,0)$. Algorithm I does not exhibit such behavior. The sequence of iterates moves toward the solution from the very first step, and is not attracted to the origin because the objective function influences the choice of search direction. As shown in Table 2, the linearized 
constraints are never satisfied (an LP is solved at every iteration) but the algorithm finds that the penalty $\pi=1$ is adequate to enforce progress. (No LP is solved in the last iteration, because the optimality stopping test is satisfied at that point). Thus, although the linear feasibility subproblem (2.8) of Algorithm I has some of the flavor of a feasibility restoration phase, it is only used to determine the penalty parameter and not to compute iterates, which is beneficial in this example.

Table 2: Output for Example 2.

\begin{tabular}{|c|c|c|c|c|c|c|c|c|c|c|}
\hline it & $\pi_{k}$ & $\Delta_{k-1}$ & QPs & LPs & $x_{1}$ & $x_{2}$ & KKT $(x)$ & feas $(x)$ & $f(x)$ & $\phi_{\pi_{+}}(x)$ \\
\hline \hline 0 & 1 & & & & 1 & 0 & $2.00 \mathrm{E}+00$ & $2.00 \mathrm{E}+00$ & $1.00 \mathrm{E}+00$ & $3.00 \mathrm{E}+00$ \\
\hline 1 & 1 & $1.00 \mathrm{E}+00$ & 1 & 1 & 0.6667 & 0.6667 & $6.67 \mathrm{E}-01$ & $7.41 \mathrm{E}-01$ & $1.11 \mathrm{E}-01$ & $8.52 \mathrm{E}-01$ \\
\hline 2 & 1 & $1.33 \mathrm{E}+00$ & 1 & 1 & 0.4444 & 0.8736 & $3.95 \mathrm{E}-01$ & $2.85 \mathrm{E}-01$ & $1.60 \mathrm{E}-02$ & $3.01 \mathrm{E}-01$ \\
\hline 3 & 1 & $4.44 \mathrm{E}-01$ & 1 & 1 & 0.2222 & 0.952 & $2.59 \mathrm{E}-01$ & $6.04 \mathrm{E}-02$ & $2.30 \mathrm{E}-03$ & $6.27 \mathrm{E}-02$ \\
\hline 4 & 1 & $4.44 \mathrm{E}-01$ & 1 & 1 & 0.1111 & 1 & $6.48 \mathrm{E}-02$ & $1.37 \mathrm{E}-02$ & $0.00 \mathrm{E}+00$ & $1.37 \mathrm{E}-02$ \\
\hline 5 & 1 & $2.22 \mathrm{E}-01$ & 1 & 1 & 0.0556 & 1 & $1.62 \mathrm{E}-02$ & $3.26 \mathrm{E}-03$ & $4.93 \mathrm{E}-32$ & $3.26 \mathrm{E}-03$ \\
\hline 6 & 1 & $1.11 \mathrm{E}-01$ & 1 & 1 & 0.0278 & 1 & $4.05 \mathrm{E}-03$ & $7.93 \mathrm{E}-04$ & $4.93 \mathrm{E}-32$ & $7.93 \mathrm{E}-04$ \\
\hline 7 & 1 & $5.56 \mathrm{E}-02$ & 1 & 1 & 0.0139 & 1 & $1.01 \mathrm{E}-03$ & $1.96 \mathrm{E}-04$ & $1.23 \mathrm{E}-32$ & $1.96 \mathrm{E}-04$ \\
\hline 8 & 1 & $2.78 \mathrm{E}-02$ & 1 & 1 & 0.0069 & 1 & $2.53 \mathrm{E}-04$ & $4.86 \mathrm{E}-05$ & $0.00 \mathrm{E}+00$ & $4.86 \mathrm{E}-05$ \\
\hline 9 & 1 & $1.39 \mathrm{E}-02$ & 1 & 1 & 0.0035 & 1 & $6.33 \mathrm{E}-05$ & $1.21 \mathrm{E}-05$ & $4.93 \mathrm{E}-32$ & $1.21 \mathrm{E}-05$ \\
\hline 10 & 1 & $6.94 \mathrm{E}-03$ & 1 & 1 & 0.0017 & 1 & $1.58 \mathrm{E}-05$ & $3.02 \mathrm{E}-06$ & $4.93 \mathrm{E}-32$ & $3.02 \mathrm{E}-06$ \\
\hline 11 & 1 & $3.47 \mathrm{E}-03$ & 1 & 1 & 0.0009 & 1 & $3.96 \mathrm{E}-06$ & $7.54 \mathrm{E}-07$ & $1.23 \mathrm{E}-32$ & $7.54 \mathrm{E}-07$ \\
\hline 12 & 1 & $1.74 \mathrm{E}-03$ & 1 & 0 & 0.0009 & 1 & $8.48 \mathrm{E}-07$ & $7.54 \mathrm{E}-07$ & $1.23 \mathrm{E}-32$ & $7.54 \mathrm{E}-07$ \\
\hline
\end{tabular}

Example 3. The following problem belongs to the class of mathematical programs with complementarity constraints (MPCCs). These problems have received much attention in recent years because of their many practical applications [12]; they can be challenging to solve because MFCQ is violated at every feasible point. The problem is given by

$$
\begin{aligned}
\operatorname{minimize} x_{1}+x_{2} & \\
\text { subject to } x_{2}^{2}-1 & \geq 0, \\
x_{1} x_{2} & \leq 0, \\
x_{1} \geq 0, x_{2} & \geq 0 .
\end{aligned}
$$

The solution is $x_{*}=(0,1)$ and is a strongly stationary point, which in the context of this paper means that there is a finite value of the penalty parameter $\pi_{*}$ such that $x_{*}$ is a stationary point for the penalty function $\phi_{\pi}(x)$, for all $\pi \geq \pi_{*}$. Equivalently, there exist multipliers at $x_{*}$ that satisfy the KKT conditions for (2.1) (although these multipliers are not unique; in fact the set of multipliers is unbounded). Fletcher et al. [16] show that the linearization of the constraints of this problem is inconsistent for any point of the form $(\epsilon, 1-\delta)$, with $\epsilon, \delta>0$.

In the results reported in Table 3 , the starting point was chosen as $(0.1,0.9)$. At the first iteration, the linearized constraints are not satisfied; the search direction computed with the initial penalty parameter satisfies condition (2.12), and the penalty parameter is not increased. At the second iteration, the search direction violates the linearized constraints, and the LP subproblem indicates that the linearized constraints can be satisfied. The 
Table 3: Output for Example 3.

\begin{tabular}{|c|c|c|c|c|c|c|c|c|c|c|}
\hline it & $\pi_{k}$ & $\Delta_{k-1}$ & QPs & LPs & $x_{1}$ & $x_{2}$ & KKT $(x)$ & feas $(x)$ & $f(x)$ & $\phi_{\pi_{+}}(x)$ \\
\hline \hline 0 & 1 & & & & $1.00 \mathrm{E}-01$ & $9.00 \mathrm{E}-01$ & $1.70 \mathrm{E}+00$ & $2.80 \mathrm{E}-01$ & $1.00 \mathrm{E}+00$ & $1.28 \mathrm{E}+00$ \\
\hline 1 & 1 & $1.00 \mathrm{E}+00$ & 1 & 1 & $-1.17 \mathrm{E}-02$ & $1.01 \mathrm{E}+00$ & $3.08 \mathrm{E}-01$ & $1.17 \mathrm{E}-02$ & $9.94 \mathrm{E}-01$ & $1.01 \mathrm{E}+00$ \\
\hline 2 & 1 & $2.23 \mathrm{E}-01$ & 3 & 1 & $-6.42 \mathrm{E}-17$ & $1.01 \mathrm{E}+00$ & $1.00 \mathrm{E}+00$ & $6.42 \mathrm{E}-17$ & $1.01 \mathrm{E}+00$ & $1.01 \mathrm{E}+00$ \\
\hline 3 & 100 & $2.35 \mathrm{E}-02$ & 1 & 0 & $-1.28 \mathrm{E}-16$ & $1.00 \mathrm{E}+00$ & $4.82 \mathrm{E}-01$ & $1.28 \mathrm{E}-16$ & $1.00 \mathrm{E}+00$ & $1.00 \mathrm{E}+00$ \\
\hline 4 & 100 & $1.11 \mathrm{E}-02$ & 1 & 0 & $-2.57 \mathrm{E}-16$ & $1.00 \mathrm{E}+00$ & $3.07 \mathrm{E}-05$ & $2.57 \mathrm{E}-16$ & $1.00 \mathrm{E}+00$ & $1.00 \mathrm{E}+00$ \\
\hline 5 & 100 & $1.00 \mathrm{E}-03$ & 1 & 0 & $-2.57 \mathrm{E}-16$ & $1.00 \mathrm{E}+00$ & $2.36 \mathrm{E}-10$ & $2.57 \mathrm{E}-16$ & $1.00 \mathrm{E}+00$ & $1.00 \mathrm{E}+00$ \\
\hline
\end{tabular}

penalty parameter needs to be increased twice so that the solution of the QP satisfies the linearized constraints. The new iterate is feasible and from that point on, the iterates converge quadratically to the solution.

Several specialized methods have been developed in recent years that exploit the structure of MPCCs (see e.g. [2, 3, 11, 16, 22, 23, 24, 27]. In these methods, the complementarity constraints must be singled out and relaxed (or penalized). Algorithm I is, in contrast, a general-purpose nonlinear programming solver that treats MPCCs as any other problem.

Example 4. MFCQ is also violated in a subset of the feasible region in the class of switch-off problems, also known as problems with vanishing constraints; see Achtziger and Kanzow [1]. An instance of such problems is

$$
\begin{aligned}
\operatorname{minimize} 2\left(x_{1}+x_{2}\right) & \\
\text { subject to } x_{1} & \geq 0, \\
x_{1} x_{2} & \geq 0, \\
x_{2} & \geq-1,
\end{aligned}
$$

which has a unique solution at $x_{*}=(0,-1)$. The feasible region is the union of the first quadrant, where the constraints are regular (except at the origin), and a portion of the negative $x_{2}$-axis, where MFCQ is violated. The performance of Algorithm I, starting at the origin, is summarized in Table 4.

Table 4: Output for Example 4.

\begin{tabular}{|c|c|c|c|c|c|c|c|c|c|c|}
\hline it & $\pi_{k}$ & $\Delta_{k-1}$ & QPs & LPs & $x_{1}$ & $x_{2}$ & KKT $(x)$ & feas $(x)$ & $f(x)$ & $\phi_{\pi_{+}}(x)$ \\
\hline \hline 0 & 1 & & & & 0 & 0 & $1.00 \mathrm{E}+00$ & $0.00 \mathrm{E}+00$ & $0.00 \mathrm{E}+00$ & $0.00 \mathrm{E}+00$ \\
\hline 1 & 1 & $1.00 \mathrm{E}+00$ & 2 & 1 & 0 & -1 & $9.00 \mathrm{E}+00$ & $0.00 \mathrm{E}+00$ & $-2.00 \mathrm{E}+00$ & $-2.00 \mathrm{E}+00$ \\
\hline 2 & 10 & $2.00 \mathrm{E}+00$ & 1 & 0 & 0 & -1 & $2.66 \mathrm{E}-15$ & $0.00 \mathrm{E}+00$ & $-2.00 \mathrm{E}+00$ & $-2.00 \mathrm{E}+00$ \\
\hline
\end{tabular}

At the starting point, the direction obtained by solving subproblem (2.7) is given by $d=(-1,-1)$ and leads away from the feasible region. Algorithm I, however, discards this direction because it does not satisfy the linearization of the constraints, which are satisfiable. The penalty parameter is increased from 1 to 10 , and the new search direction $d=(0,-1)$ not only satisfies the linearized constraints but leads straight to the solution of problem (4.4). The second iteration is required simply to compute the optimal multipliers. 
This example shows how a prompt identification of an inadequate penalty parameter can save a great deal of computational work. Classical penalty methods would not increase the penalty parameter at the first iteration and would follow the initial direction $(-1,-1)$. The penalty function $\phi_{\pi}$ is unbounded below along this direction and a classical algorithm would generate a series of iterates with decreasing values of $x_{1}$ until the algorithm detects that the iterates appear to be diverging. Not only would those iterations be wasted, but extra effort would be required to return to the vicinity of the solution.

Example 5. The following infeasible problem has been studied by Burke and Han [5] and Chen and Goldfarb [10]:

$$
\begin{aligned}
\operatorname{minimize} x & \\
\text { subject to } x^{2}+1 & \leq 0, \\
x & \leq 0 .
\end{aligned}
$$

Chen and Goldfarb report that, starting from $x_{1}=10$, their method converges to the infeasible stationary point $x_{*}=0$ after 50 iterations, with a final penalty parameter of $\pi \simeq 10^{6}$. Algorithm I converges to that infeasible stationary point in 3 iterations; see Table 5 .

It may seem surprising that the final penalty parameter reported in the Table 5 is only 10, given that our analysis suggests that the penalty parameter will tend to infinity in the infeasible case. We note, however, that $\pi=10$ is the penalty at the beginning of iteration 3 and that Algorithm I drives the penalty parameter to infinity in Step 4. It does so, while staying at the current iterate, and once it detects that the problem is locally infeasible, it stops.

Table 5: Output for Example 5.

\begin{tabular}{|c|c|c|c|c|c|c|c|c|c|}
\hline it & $\pi_{k}$ & $\Delta_{k-1}$ & QPs & LPs & $x$ & KKT $(x)$ & feas $(x)$ & $f(x)$ & $\phi_{\pi_{+}}(x)$ \\
\hline \hline 0 & 1 & & & & 10 & $1.01 \mathrm{E}+02$ & $1.11 \mathrm{E}+02$ & $1.00 \mathrm{E}+01$ & $1.21 \mathrm{E}+02$ \\
\hline 1 & 1 & $1.00 \mathrm{E}+00$ & 1 & 1 & 4.95 & $2.55 \mathrm{E}+01$ & $3.05 \mathrm{E}+01$ & $4.95 \mathrm{E}+00$ & $3.54 \mathrm{E}+01$ \\
\hline 2 & 1 & $1.01 \mathrm{E}+01$ & 2 & 1 & 0 & $4.01 \mathrm{E}+00$ & $1.00 \mathrm{E}+00$ & $8.88 \mathrm{E}-16$ & $1.00 \mathrm{E}+01$ \\
\hline 3 & 10 & $9.90 \mathrm{E}+00$ & 1 & 1 & 0 & $1.00 \mathrm{E}+00$ & $1.00 \mathrm{E}+00$ & $8.88 \mathrm{E}-16$ & $1.00 \mathrm{E}+01$ \\
\hline
\end{tabular}

More results obtained with our MATLAB implementation of Algorithm I are reported in [24]. The test set used in those experiments includes both regular problems and problems that are infeasible or do not satisfy constraint qualifications. The results in [24] indicate that Algorithm I is efficient on problems that do not require regularization because condition (2.11) guarantees that a pure SQP step is used whenever the linearized constraints can be satisfied in a neighborhood of the current iterate. Therefore, for regular problems, Algorithm I performs very similarly to a classical SQP method, except that a few extra QPs and LPs are solved when the initial penalty parameter is too small. On the other hand, Algorithm I is more robust and efficient than a classical SQP method on problems 
(such as those in the Examples 1-5) that require regularization. We believe that by using warm starts, the cost of solving the additional QPs is not significant, but a careful sparse implementation of Algorithm I is needed to measure the computational cost of various components of the iteration.

\section{$5 \quad$ Final Remarks}

In this paper we have proposed a line search SQP penalty method for nonlinear programming. The method updates the penalty parameter dynamically using an extension of the steering rules described in [8] to the line search setting. The resulting algorithm is robust and its global convergence properties are as strong as those of trust region methods. Specifically, we have proved that under common assumptions all limit points of the sequence of iterates are either KKT points, infeasible stationary points, or points of MFCQ failure. This fact shows that use of exact penalties, together with a positive definite Hessian approximation, has a regularizing effect similar to a trust region.

\section{References}

[1] W. Achtziger and C. Kanzow. Mathematical programs with vanishing constraints: optimality conditions and constraint qualifications. Mathematical Programming, 114(1):6999, 2008.

[2] M. Anitescu. Global convergence of an elastic mode approach for a class of Mathematical Programs with Complementarity Constraints. SIAM Journal on Optimization, 16(1):120-145, 2005.

[3] H.Y. Benson, A. Sen, D.F. Shanno, and R.J. Vanderbei. Interior-point algorithms, penalty methods and equilibrium problems. Computational Optimization and Applications, 34(2):155-182, 2006.

[4] J. M. Borwein and A. S. Lewis. Convex Analysis and Nonlinear Optimization: Theory and Examples. Springer Verlag, New York, 2000.

[5] J. V. Burke and S. P. Han. A robust sequential quadratic-programming method. Mathematical Programming, 43(3):277-303, 1989.

[6] R. H. Byrd, N. I. M. Gould, J. Nocedal, and R. A. Waltz. An algorithm for nonlinear optimization using linear programming and equality constrained subproblems. Mathematical Programming, Series B, 100(1):27-48, 2004.

[7] R. H. Byrd, N. I. M. Gould, J. Nocedal, and R. A. Waltz. On the convergence of successive linear-quadratic programming algorithms. SIAM Journal on Optimization, 16(2):471-489, 2006.

[8] R. H. Byrd, J. Nocedal, and R. A. Waltz. Steering exact penalty methods. Optimization Methods and Software, 23(2), 2008. 
[9] R. H. Byrd, J. Nocedal, and R.A. Waltz. KNITRO: An integrated package for nonlinear optimization. In G. di Pillo and M. Roma, editors, Large-Scale Nonlinear Optimization, pages 35-59. Springer, 2006.

[10] L. Chen and D. Goldfarb. Interior-point $\ell_{2}$ penalty methods for nonlinear programming with strong global convergence properties. Mathematical Programming, 108(1):1-36, 2006.

[11] De Miguel, A.V., M.P. Friedlander, F.J. Nogales, and S. Scholtes. A two-sided relaxation scheme for mathematical programs with equilibriums constraints. SIAM Journal on Optimization, 16(1):587-609, 2005.

[12] M. C. Ferris and J. S. Pang. Engineering and economic applications of complementarity problems. SIAM Review, 39(4):669-713, 1997.

[13] R. Fletcher. Practical Methods of Optimization. J. Wiley and Sons, Chichester, England, second edition, 1987.

[14] R. Fletcher and S. Leyffer. User manual for filterSQP. Technical Report NA/181, Dundee, Scotland, 1998.

[15] R. Fletcher and S. Leyffer. Nonlinear programming without a penalty function. Mathematical Programming, 91:239-269, 2002.

[16] R. Fletcher, S. Leyffer, D. Ralph, and S. Scholtes. Local convergence of SQP methods for mathematical programs with equilibrium constraints. SIAM Journal on Optimization, 17(1):259-286, 2006.

[17] R. Fletcher, S. Leyffer, and Ph. L. Toint. On the global convergence of a filter-SQP algorithm. SIAM Journal on Optimization, 13(1):44-59, 2002.

[18] P. E. Gill, W. Murray, and M. A. Saunders. SNOPT: An SQP algorithm for large-scale constrained optimization. SIAM Journal on Optimization, 12:979-1006, 2002.

[19] P. E. Gill, W. Murray, and M. H. Wright. Practical Optimization. Academic Press, London, 1981.

[20] N. I. M. Gould and D. P. Robinson. A second derivative SQP method with imposed descent. Technical Report 08/09, Oxford University Computing Laboratory, 2008.

[21] S. P. Han and O. L. Mangasarian. Exact penalty functions in nonlinear programming. Mathematical Programming, 17(1):251-269, 1979.

[22] S. Leyffer, G. López-Calva, and J. Nocedal. Interior methods for mathematical programs with complementarity constraints. SIAM Journal on Optimization, 17(1):52-77, 2006.

[23] X. Liu and J. Sun. Generalized stationary points and an interior-point method for mathematical programs with equilibrium constraints. Mathematical Programming, 101(1):231-261, 2004. 
[24] G. López-Calva. Exact-Penalty Methods for Nonlinear Programming. PhD thesis, Industrial Engineering \& Management Sciences, Northwestern University, Evanston, IL, USA, 2005.

[25] O. L. Mangasarian and S. Fromovitz. The Fritz John necessary optimality conditions in the presence of equality and inequality constraints. Journal of Mathematical Analysis and Applications, 17:37-47, 1967.

[26] J. Nocedal and S. J. Wright. Numerical Optimization. Springer Series in Operations Research. Springer, second edition, 2006.

[27] Raghunathan, A. and L. T. Biegler. An interior point method for mathematical programs with complementarity constraints (MPCCs). SIAM Journal on Optimization, 15(3):720-750, 2005.

[28] A. Ruszcyynski. Nonliner Optimization. Princeton University Press, 2006.

[29] A. Wächter and L. T. Biegler. Failure of global convergence for a class of interior point methods for nonlinear programming. Mathematical Programming, 88(3):565-574, 2000.

[30] R. A. Waltz and T. D. Plantenga. Knitro 5.0 User's Manual. Technical report, Ziena Optimization, Inc., Evanston, IL, USA, February 2006. 